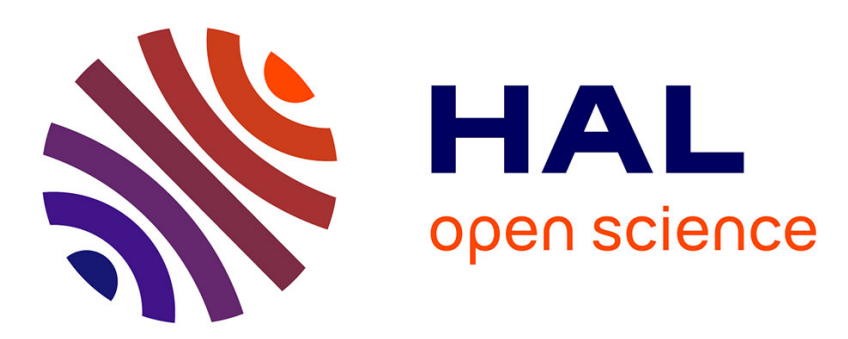

\title{
Joint Operator Pricing and Network Selection Game in Cognitive Radio Networks: Equilibrium, System Dynamics and Price of Anarchy
}

Jocelyne Elias, Fabio Martignon, Lin Chen, Eitan Altman

\section{- To cite this version:}

Jocelyne Elias, Fabio Martignon, Lin Chen, Eitan Altman. Joint Operator Pricing and Network Selection Game in Cognitive Radio Networks: Equilibrium, System Dynamics and Price of Anarchy. IEEE Transactions on Vehicular Technology, 2013, 62 (9), pp.4576-4589. 10.1109/TVT.2013.2264294 . hal00913254

\section{HAL Id: hal-00913254 \\ https://hal.inria.fr/hal-00913254}

Submitted on 8 Dec 2013

HAL is a multi-disciplinary open access archive for the deposit and dissemination of scientific research documents, whether they are published or not. The documents may come from teaching and research institutions in France or abroad, or from public or private research centers.
L'archive ouverte pluridisciplinaire HAL, est destinée au dépôt et à la diffusion de documents scientifiques de niveau recherche, publiés ou non, émanant des établissements d'enseignement et de recherche français ou étrangers, des laboratoires publics ou privés. 


\title{
Joint Operator Pricing and Network Selection Game in Cognitive Radio Networks: Equilibrium, System Dynamics and Price of Anarchy
}

Jocelyne Elias, Université Paris Descartes, E-mail: jocelyne.elias@parisdescartes.fr Fabio Martignon, Lin Chen, Université Paris-Sud, E-mail: \{fabio.martignon, lin.chen\}@lri.fr Eitan Altman, INRIA Sophia Antipolis, France, E-mail: Eitan.Altman@sophia.inria.fr

\begin{abstract}
This paper addresses the joint pricing and network selection problem in cognitive radio networks. The problem is formulated as a Stackelberg game where first the Primary and Secondary operators set the network subscription price to maximize their revenue. Then, users perform the network selection process, deciding whether to pay more for a guaranteed service, or use a cheaper, best-effort secondary network, where congestion and low throughput may be experienced.

We derive optimal stable price and network selection settings. More specifically, we use the Nash equilibrium concept to characterize the equilibria for the price setting game. On the other hand, a Wardrop equilibrium is reached by users in the network selection game, since in our model a large number of users must determine individually the network they should connect to. Furthermore, we study network users' dynamics using a population game model, and we determine its convergence properties under replicator dynamics, a simple yet effective selection strategy.

Numerical results demonstrate that our game model captures the main factors behind cognitive network pricing and network selection, thus representing a promising framework for the design and understanding of cognitive radio systems.

Index Terms: - Cognitive Radio Networks, Pricing, Network Selection, Stackelberg Game, Population Game Model, Replicator Dynamics.
\end{abstract}

\section{INTRODUCTION}

Cognitive radio networks (CRNs), also referred to as $x G$ networks, are envisioned to deliver high bandwidth to mobile users via heterogeneous wireless architectures and dynamic spectrum access tech- 
niques [1], [2]. In CRNs, a Primary (or licensed) User (PU) has a license to operate in a certain spectrum band; his access is generally controlled by the Primary Operator (PO) and should not be affected by the operations of any other unlicensed user. On the other hand, the Secondary Operator (SO) has no spectrum license; therefore, Secondary Users (SUs) must implement additional functionalities to share the licensed spectrum band without interfering with primary users.

In this work, we consider a cognitive radio scenario which consists of primary and secondary networks, as well as a large set of cognitive users, and we focus on a fundamental issue concerning such systems, i.e. whether it is better for a CR user to act as a primary user, paying the Primary operator for costlier, dedicated network resources with Quality of Service guarantees, or act as a secondary user (paying the Secondary operator), sharing the spectrum holes left available by licensed users and facing lower costs with degraded performance guarantees. At the same time, we consider the pricing problem of both Primary and Secondary operators, who compete with each other, setting access prices to maximize their revenues.

The joint pricing and cognitive radio network selection problem is modeled as a Stackelberg (leaderfollower) game, where first the Primary and Secondary operators set their access prices in order to maximize their revenues. In this regard, we study both practical cases where (1) the Primary and Secondary operators fix access prices at the same time, and (2) the Primary operator exploits his dominant position by playing first, anticipating the choices of the Secondary operator. Then, network users react to the prices set by the operators, choosing which network they should connect to, therefore acting either like primary or secondary users.

The solution provides an insight on how rational users will distribute among existing access solutions (higher-price primary networks vs. lower-price secondary networks), i.e., the proportion of players who choose different strategies.

We adopt a fluid queue approximation approach (as in [3], [4], [5], [6], [7]) to study the steadystate performance of these users, focusing on delay as QoS metric. Besides considering static traffic equilibrium settings, we further formulate the network selection process of cognitive radio users as a population game [8], which provides a powerful framework for characterizing the strategic interactions among large numbers of agents, whose behavior is modeled as a dynamic adjustment process. More specifically, we study the cognitive users' behavior according to replicator dynamics [8], [9], since such users adapt their choices and strategies based on the observed network state. 
We provide equilibrium and convergence properties of the proposed game, and derive optimal stable price and network selection settings.

More specifically, we use the Nash equilibrium concept to characterize the equilibria of the pricing game between a finite number of decision makers (viz., the Primary and Secondary operators). In addition to that, we further determine the Wardrop equilibrium for the network selection game, in which a large number of users must choose individually the network they should connect to. Such equilibrium is characterized by two properties, namely traffic equilibrium (the total costs perceived by users on all used networks are equal) and system optimum principle (the average delay/cost is minimum) [10].

Numerical results obtained in different network scenarios illustrate that our game captures the main factors behind cognitive network pricing and selection, thus representing a promising framework for the design and performance evaluation of cognitive radio systems.

In summary, in an effort to understand the pricing and networking selection issues that characterize CRNs, our work makes the following contributions:

- the proposition of a novel game theoretical model where Primary and Secondary operators set access prices, and users select which network to connect to, based both on the total delay and the experienced cost.

- The computation of equilibrium points for our game, as well as relevant performance metrics, including the Price of Anarchy and the Price of Stability.

- The analysis of a dynamic model, based on population games, which further illustrates how players converge to the equilibrium in a dynamic context under an easily implementable, distributed strategy (viz., replicator dynamics), along with formal, detailed proofs of its convergence.

The rest of this paper is organized as follows: related work is reviewed in Section II. The network model for the proposed joint pricing and network selection game is described in Section III; the equilibrium points of such game, as well as its Price of Anarchy and Price of Stability, are derived in Sections IV and $\mathrm{V}$, respectively. The dynamic network selection model, based on population games and replicator dynamics, is presented in Section VI, and its convergence properties are demonstrated in Section VII. Numerical results are discussed in Section VIII, while Section IX concludes this work. 


\section{RELATED WORK}

In this section, we first review the most notable works on spectrum pricing and access in cognitive radio networks [3], [4], [11], [12], [13], [14], [15], [16], [17], [18]. Then, we discuss relevant works that use evolutionary games to study the users behavior in CR as well as in heterogeneous wireless networks [19], [20], [21], [22], [23].

In [11], the authors provide a systematic overview on CR networking and communications, by looking at the key functions of the physical, MAC and network layers involved in a CR design, and by studying how these layers are crossly related. In [3], the authors consider the decision-making process of SUs

who have the choice of either acquiring a dedicated spectrum (paying a price) or using the primary user band for free, and they characterize the resulting Nash equilibrium for the single-band case. This work differs from ours in two main aspects: 1) the CR users already arrive at the system as secondary or primary ones; SUs have the choice between dedicated or PU band, and 2) the users' behavior is studied based on queueing theory. The work in [4] considers a CRN where multiple secondary users (SUs) contend for spectrum usage, using random access, over available primary user channels, focusing on SUs' queueing delay performance. A fluid queue approximation approach is adopted to study the steady-state delay performance of SUs. In [12], the authors analyze the price competition between PUs who can lease out their unused bandwidth to secondaries in exchange for a fee, considering bandwidth uncertainty and spatial reuse. The problem of dynamic spectrum leasing in a secondary market of CRNs is considered in [14], where secondary service providers lease spectrum from spectrum brokers to provide service to SUs.

Recent works have considered evolutionary games to study the users' behavior in cognitive radio and heterogeneous wireless networks [19], [20], [21], [22], [23].

In [19], the authors use evolutionary game theory to investigate the dynamics of user behavior in heterogeneous wireless access networks (i.e., WMANs, cellular networks, and WLANs). The evolutionary game solution is compared to the Nash equilibrium, and a set of algorithms (i.e., population evolution and reinforcement learning algorithms) are proposed to implement the evolutionary network selection game model. In [20], the dynamics of a multiple-seller, multiple-buyer spectrum trading market is modeled as an evolutionary game, in which PUs want to sell and SUs want to buy spectrum opportunities. Secondary users evolve over time, buying the spectrum opportunities that optimize their performance in terms of transmission rate and price. In [21], the authors propose a distributed framework for spectrum access, with 
and without complete network information (i.e., channel statistics and user selections). In the first case, an evolutionary game approach is proposed, in which each SU compares its payoff with the system average payoff to evolve its spectrum access decision over time. For the incomplete information case, a learning mechanism is proposed, in which each SU estimates its expected throughput locally and learns to adjust its channel selection strategy adaptively. The problem of opportunistic spectrum access in CSMA/CA-based cognitive radio networks is also addressed in [22] from an evolutionary game theoretic angle.

In our preliminary works [24], [25], we addressed the pricing and network selection problems in cognitive radio networks. However, in [24], we assumed that the PO and SO use separate frequency bands, which greatly simplifies the problem, and we did not study the impact of the order in which operators set prices on the quality of the reached equilibria. The work in [25] differs from the one presented here in that it considered uniquely Primary operators, and a finite set of SUs, which are characterized by elastic traffic demands that can be transmitted over one or multiple frequency spectra.

Unlike previous works, which study the interaction between two well-defined sets of users (primary and secondary ones) who already performed the choice of using the primary or the secondary network, our paper tackles a fundamental issue in CRNs. In fact, we model the users' decision process that occurs before such users enter the CRN, thus assessing the economic interest of deploying secondary (xG) networks. Such choice depends on the trade-off between cost and performance guarantees in such networks. At the same time, we derive the optimal price setting for both Primary and Secondary operators that play before network users, in order to maximize their revenue. We use enhanced game theoretical tools, derived from population game theory, to model the network selection dynamics, providing convergence conditions and equilibrium settings.

\section{NETwORK MODEL}

We now detail the network model, which is illustrated in Figure 1: a cognitive radio wireless system which consists of a secondary $(\mathrm{xG})$ network that coexists with a primary network at the same location and on the same spectrum band.

We consider an overlay model (focusing on the "interference avoidance" approach [26], [27] to cognitive radio) as in [3], [20], [28], where Secondary Users periodically sense the radio spectrum, intelligently detect occupancy in the different frequency bands and then opportunistically communicate over the spectrum holes left available by Primary Users, thus avoiding interference with active primary users. In 


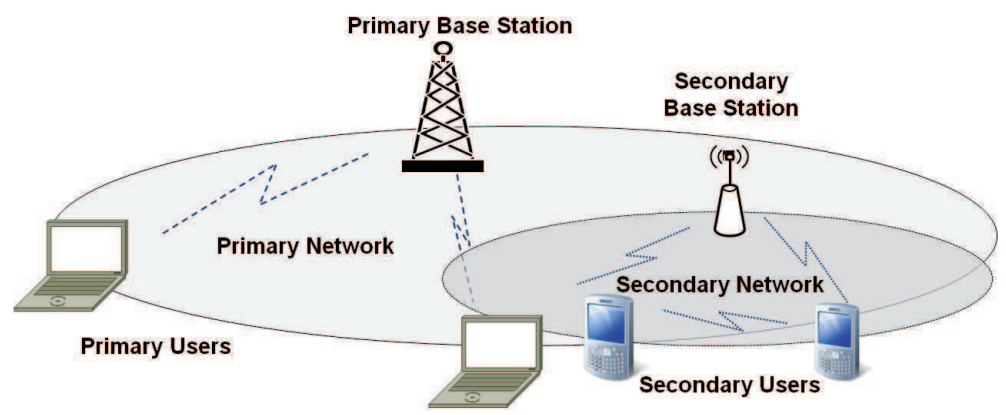

Fig. 1. CRN scenario with a primary network and a secondary $(\mathrm{xG})$ network. Arriving users must decide whether to join the primary network, paying a subscription fee $\left(p_{1}\right)$ for guaranteed QoS, or the $\mathrm{xG}$ network (which has a lower subscription cost, $p_{2}<p_{1}$, and less performance guarantees), based on the expected cost and congestion levels.

other words, our model is an overlay CR where secondary users opportunistically access primary users' spectrum only when it is not occupied. As in [3], we further consider perfect primary user detection at the secondary users and zero interference tolerance at each of the primary and secondary users.

We assume that users arrive at this system following a Poisson process with rate $\lambda$, and the maximum achievable transmission rate of the wireless channel (licensed to the PO and opportunistically used by the SO) is denoted by $C$. The total traffic $\lambda$ admitted in the network must not exceed its capacity $C$; this can be obtained, for example, using admission control techniques, which are out of the scope of this paper. All these assumptions are commonly adopted in several recent works like [4], [5], [6], [7].

Each arriving user must choose whether to join the primary network (paying a higher subscription cost) or the $\mathrm{xG}$ one (which has a lower subscription cost), based on criteria to be specified below, i.e., a combination of cost and QoS (service time/latency).

Finally, let us denote by $\lambda_{P}$ the overall transmission rate of primary users (i.e., those who choose the primary network) and by $\lambda_{S}$ the rate of secondary users, so that $\lambda=\lambda_{P}+\lambda_{S}$. Table I summarizes the basic notation used in our game model.

We now define users' cost functions as well as the utility functions of Primary and Secondary operators. We assume that the total cost incurred by a network user is a combination of the service time (delay, or latency) experienced in the network, and the cost for the player to access such network.

We underline that a similar model is used in [3], where the average cost incurred by a Secondary User (SU) consists of two components: (1) the price $(\tilde{C})$ of the dedicated spectrum band, and (2) an average delay cost $\left(\frac{1}{\mu}\right)$, where $\mu$ is the service time. The average delay cost is weighted by a parameter $\alpha$, which represents the delay vs. monetary cost tradeoff of the SUs. To further support our choice, another similar 
TABLE I

BASIC NOTATION

\begin{tabular}{|l|l|}
\hline$\lambda$ & Total traffic accepted in the network \\
\hline$C$ & Wireless channel capacity (Maximum achievable transmission rate of the wireless channel) \\
\hline$\alpha$ & Weighting parameter of delay with respect to access cost \\
\hline$\lambda_{P}$ & Total traffic transmitted by Primary Users \\
\hline$\lambda_{S}$ & Total traffic transmitted by Secondary Users \\
\hline$X_{P}$ & Fraction of Primary Users \\
\hline$X_{S}$ & Fraction of Secondary Users \\
\hline$p_{1}, p_{2}$ & Price charged by the PO and the SO, respectively, to access its services \\
\hline$K$ & Constant, velocity of convergence \\
\hline
\end{tabular}

model is considered by Anshelevich et al. in [29] for a different networking context. The authors set the player's cost for using an edge $e$ in the network as a combination of a cost function $c_{e}(x)$ and a latency function $d_{e}(x)$; the goal of each user in such game is to minimize the sum of his cost and latency. The same model is also used in [30]. Finally, note that in [19] the authors consider two components, namely throughput (the allocated capacity to a player, which is obviously related to the delay experienced by such user) and the corresponding price (see equations (2) and (3) in [19]).

In this work, we consider a fluid queue approximation approach, which permits to study the steady-state delay performance of both PUs and SUs. To this aim, and without loss of generality, we assume that the wireless channel is modeled as a $\mathrm{M}|\mathrm{M}| 1$ queue, with service rate $C$ and arrival rate $\lambda$. Recall that both the primary and secondary networks operate on the same channel; the Primary and Secondary operators fix the prices $p_{1}$ and $p_{2}$, respectively, for accessing their services. Therefore, the total cost perceived by primary users is given by:

$$
\operatorname{Cost}_{P U}=\frac{\alpha}{C-\lambda_{P}}+p_{1}
$$

where parameter $\alpha$ weights the relative importance of the experienced delay with respect to the access cost. Note that primary users are affected exclusively by the traffic transmitted by primary users $\left(\lambda_{P}\right)$, and not by the traffic of secondary users $\left(\lambda_{S}\right)$, since usually, in a cognitive radio network, primary users have strict priority over secondary users; these latter must therefore implement spectrum sensing and spectrum handover strategies to avoid any interference towards primary users, and can transmit only in the spectrum holes left unoccupied by these ones.

As mentioned previously, we consider perfect primary user detection at the secondary users and zero 
interference tolerance at each of the primary and secondary users.

For this reason, secondary users' performance is affected by the whole traffic, transmitted by both primary and secondary users; such users are characterized by the following cost function:

$$
\operatorname{Cost}_{S U}=\frac{\alpha}{C-\left(\lambda_{P}+\lambda_{S}\right)}+p_{2}=\frac{\alpha}{C-\lambda}+p_{2}
$$

As for operators' utilities, they correspond to the total revenue obtained by pricing users. As a consequence, the Primary operator's utility function is expressed as follows:

$$
U_{P}=p_{1} \lambda_{P}
$$

Correspondingly, the Secondary operator's utility function is:

$$
U_{S}=p_{2} \lambda_{S}=p_{2}\left(\lambda-\lambda_{P}\right)
$$

To summarize, network users minimize the perceived cost, which is expressed as $\operatorname{Cost}_{P U}=\frac{\alpha}{C-\lambda_{P}}+p_{1}$ (see equation (1)) if they choose the primary network, and $\operatorname{Cost}_{S U}=\frac{\alpha}{C-\lambda}+p_{2}$ (see equation (2)) if they act as secondary users. As for Primary/Secondary operators, they try to maximize the total revenue obtained by pricing primary $\left(U_{P}=p_{1} \lambda_{P}\right)$ or secondary users $\left(U_{S}=p_{2} \lambda_{S}\right)$, respectively. Users' cost functions as well as operators' utilities are also reported in Tables II and III, respectively.

TABLE II

PRIMARY AND SECONDARY USER's COST FUNCTIONS

\begin{tabular}{|l|l|}
\hline Primary User $(\mathrm{PU})$ & $\operatorname{Cost}_{P U}=\frac{\alpha}{C-\lambda_{P}}+p_{1}$ \\
\hline Secondary User $(\mathrm{SU})$ & Cost $_{S U}=\frac{\alpha}{C-\lambda}+p_{2}$ \\
\hline
\end{tabular}

TABLE III

PRIMARY AND SECONDARY OPERATOR's UTILITY FUNCTIONS

\begin{tabular}{|l|l|}
\hline Primary Operator (PO) & $U_{P}=p_{1} \lambda_{P}$ \\
\hline Secondary Operator $(\mathrm{SO})$ & $U_{S}=p_{2} \lambda_{S}$ \\
\hline
\end{tabular}

\section{EQUILIBRIUM COMPUTATION}

In this section, we derive the equilibrium points of our game, namely: (i) the equilibrium traffic sent by primary and secondary users, (ii) steady-state Primary/Secondary operator's utilities, as well as (iii) equilibrium prices set by the $\mathrm{PO} / \mathrm{SO}$. 
We consider two practical cases: (1) both operators fix their access price at the same time, trying to maximize their own revenue (Section IV-A), and (2) the PO plays before the SO, anticipating the strategy of this latter, thus exploiting his dominant position (Section IV-B). We will refer to the first case as the TOGETHER scenario, while the latter will be referred to as the BEFORE scenario. Note that when the Primary and Secondary operators play at the same time, we have a Cournot duopoly competition between such operators. However, in the original Cournot duopoly, production quantities (outputs) and prices are linear, while in this work we consider a nonlinear system which requires non-standard studies that cannot rely on existing results. On the other hand, when the Primary operator plays before the Secondary, anticipating his choices, we have a Stackelberg game model between the operators.

The Nash equilibrium concept will be used for the price setting game, since we have a finite number of decision makers, i.e., the two network operators. More precisely, a Nash Equilibrium is a set of players' (here, operators') strategies, each of which maximizes the player's revenue, and such that none of the actors has an incentive to deviate unilaterally. For this reason the corresponding network configurations are said to be stable.

On the other hand, a Wardrop equilibrium [31] is reached by CR users in the network selection game, since in our model a large number of users must determine individually the network they should connect to. Such equilibrium satisfies the two Wardrop's principles, namely traffic equilibrium (the total costs perceived by users on all used networks are equal) and system optimum principle (the average delay/cost is minimum).

Therefore, at Wardrop equilibrium, primary and secondary users will both experience the same cost, that is, $\operatorname{Cost}_{P U}=\operatorname{Cost}_{S U}$, or:

$$
\frac{\alpha}{C-\lambda_{P}}+p_{1}=\frac{\alpha}{C-\left(\lambda_{P}+\lambda_{S}\right)}+p_{2}=\frac{\alpha}{C-\lambda}+p_{2}
$$

This permits to compute the equilibrium traffic ${ }^{1}$ for the primary network as a function of the prices set by both the PO and SO:

$$
\lambda_{P}=\frac{\alpha \lambda-C(C-\lambda)\left(p_{1}-p_{2}\right)}{\alpha-(C-\lambda)\left(p_{1}-p_{2}\right)}
$$

with $0 \leq \lambda_{P} \leq \lambda$. The traffic sent by secondary users, $\lambda_{S}$, will therefore be equal to $\lambda-\lambda_{P}$. Note that,

\footnotetext{
${ }^{1}$ With a slight abuse of notation, we will denote equilibrium flows still by $\lambda_{P}$ and $\lambda_{S}$, since in the following we will almost exclusively refer to equilibrium game conditions.
} 
in order for the equilibrium condition (5) to hold and for equilibrium traffic $\lambda_{P}$ to be comprised in the $[0, \lambda]$ range, $p_{1}-p_{2}$ must satisfy the condition $p_{1}-p_{2}<\frac{\alpha \lambda}{C(C-\lambda)}$. Furthermore, since there is a unique $\lambda_{P}$ value which satisfies condition (5), such value represents the unique Wardrop equilibrium point of the network selection game.

The corresponding equilibrium utility for the PO is given by the following expression:

$$
U_{P}=p_{1} \lambda_{P}=p_{1} \cdot \frac{\alpha \lambda-C(C-\lambda)\left(p_{1}-p_{2}\right)}{\alpha-(C-\lambda)\left(p_{1}-p_{2}\right)}
$$

while the utility of the SO will be:

$$
U_{S}=p_{2} \lambda_{S}=p_{2}\left(\lambda-\lambda_{P}\right)=p_{2} \lambda+p_{2}\left[\frac{\alpha(C-\lambda)}{\alpha-(C-\lambda)\left(p_{1}-p_{2}\right)}-C\right]
$$

Hereafter we compute equilibrium prices for both our considered scenarios.

\section{A. The Primary and Secondary operators fix their prices simultaneously (TOGETHER scenario)}

In this scenario, both the Primary and Secondary operators fix their prices simultaneously, trying to maximize their own revenue. As a consequence, to maximize the utility function of the PO, it suffices to take the derivative of $U_{P}$ with respect to $p_{1}$, imposing its equality to zero:

$$
\frac{\partial U_{P}}{\partial p_{1}}=C-\frac{\alpha(C-\lambda)\left[\alpha-(C-\lambda)\left(p_{1}-p_{2}\right)\right]+\alpha(C-\lambda)^{2} p_{1}}{\left[\alpha-(C-\lambda)\left(p_{1}-p_{2}\right)\right]^{2}}=0,
$$

Hence, we can express the price $p_{1}$ as a function of $p_{2}$ :

$$
p_{1}=p_{2}+\frac{\alpha}{C-\lambda}\left\{1-\sqrt{\frac{(C-\lambda)}{\alpha C}\left[\alpha+(C-\lambda) p_{2}\right]}\right\}
$$

Similarly, the Secondary operator aims at maximizing his revenue $U_{S}$; by deriving $U_{S}$ with respect to $p_{2}$ and imposing its equality to zero, we obtain:

$$
\frac{\partial U_{S}}{\partial p_{2}}=(\lambda-C)+\frac{\alpha^{2}(C-\lambda)-\alpha(C-\lambda)^{2} p_{1}}{\left[\alpha-(C-\lambda)\left(p_{1}-p_{2}\right)\right]^{2}}=0
$$


and the expression of $p_{2}$ as a function of $p_{1}$ is given by:

$$
p_{2}=p_{1}-\frac{1}{(C-\lambda)}\left\{\alpha-\sqrt{\alpha^{2}-\alpha(C-\lambda) p_{1}}\right\} .
$$

Finally, combining expressions (10) and (12) we obtain the equilibrium price values $p_{1}$ and $p_{2}$, which are function of $\alpha, C$ and $\lambda$ :

$$
\begin{gathered}
p_{1}=\alpha \frac{\left(3 C^{2}-\lambda^{2}\right)-(C-\lambda)^{2} \sqrt{\frac{9 C-5 \lambda}{C-\lambda}}}{2(2 C-\lambda)^{2}(C-\lambda)} \\
p_{2}=\alpha \frac{C \sqrt{9 C-5 \lambda}-(3 C-2 \lambda) \sqrt{C-\lambda}}{2(2 C-\lambda)^{2} \sqrt{C-\lambda}},
\end{gathered}
$$

with $p_{1} \geq 0$ and $p_{2} \geq 0$.

\section{B. The Primary operator plays before the Secondary (BEFORE scenario)}

In this case, we have a Stackelberg game between operators, in which the Primary operator is the leader while the Secondary operator is the follower.

The PO will therefore anticipate the choice of the SO (who will set the price $p_{2}$ in order to maximize his utility), and will play his best strategy, setting the optimal value for $p_{1}$ taking into account the choice on $p_{2}$ operated by the SO.

To derive the equilibrium prices in such scenario, it suffices to take the derivative of $U_{S}$ with respect to the price $p_{2}$, obtaining $p_{2}$ in function of $p_{1}$ (see equation (12)). We next insert the expression of $p_{2}$ in (7), obtaining $U_{P}$ as a function of $p_{1}$ :

$$
U_{P}=p_{1}\left\{C+\frac{\alpha(\lambda-C)}{\sqrt{\alpha^{2}-\alpha(C-\lambda) p_{1}}}\right\}
$$

Deriving $U_{P}$ with respect to the price $p_{1}$, we obtain $C+\frac{\sqrt{\alpha}(\lambda-c)\left[2 \alpha-(C-\lambda) p_{1}\right]}{2\left[\alpha-(C-\lambda) p_{1}\right]^{3 / 2}}$; then, imposing that such derivative is null, we obtain the equilibrium value for $p_{1}$, which has the following expression:

$$
p_{1}=\frac{\alpha}{C-\lambda}\left\{1-\left(Z+\frac{h}{3}\right)^{2}\right\}
$$

where $Z=\left(\frac{h}{4}\right)^{1 / 3}\left[\left(\sqrt{1+\frac{4}{27} h^{2}}+1\right)^{2 / 3}+\left(\sqrt{1+\frac{4}{27} h^{2}}-1\right)^{2 / 3}\right]$, and $h=\frac{C-\lambda}{2 C}$. 
If we combine such expression of $p_{1}$ with (12), we obtain the equilibrium price set by the Secondary operator:

$$
p_{2}=\frac{\alpha}{C-\lambda}\left(Z+\frac{h}{3}\right)\left[1-\left(Z+\frac{h}{3}\right)\right]
$$

\section{Comments}

Note that, in both the TOGETHER and BEFORE scenarios, equilibrium prices are unique. In fact, if

we compute the second derivatives in both network scenarios $\left(\frac{\partial^{2} U_{P}}{\partial p_{1}{ }^{2}}\right.$ and $\left.\frac{\partial^{2} U_{S}}{\partial p_{2}{ }^{2}}\right)$, they are both negative for all price values in the feasible region $p_{1}-p_{2}<\frac{\alpha \lambda}{C(C-\lambda)}$. Hence, the maximums, as well as the Nash equilibrium points, are unique.

Furthermore, equilibrium prices $\left(p_{1}\right.$ and $p_{2}$ ) are directly proportional to $\alpha$, while equilibrium flows ( $\lambda_{P}$ and $\lambda_{S}$ ) are independent of $\alpha$; this can be seen by substituting, in expression (6), $p_{1}-p_{2}$, which is proportional to $\alpha$. As a consequence, operators' utilities grow proportionally to $\alpha$. All these trends will be illustrated in more detail in the Numerical Results section.

Finally, primary users' equilibrium traffic, $\lambda_{P}$, decreases with increasing $C$ values, while secondary users' traffic follows an opposite trend. As for operators' prices and utilities, they both decrease with $C$, as we will quantify in Section VIII.

\section{Price of ANARChy AND Price of Stability}

We now investigate the efficiency of the equilibria reached by operators and users in our joint pricing and network selection game, through the determination of the Price of Anarchy (PoA) and the Price of Stability (PoS). They both quantify the loss of efficiency as the ratio between the cost of a specific stable outcome/equilibrium and the cost of the optimal outcome, which could be designed by a central authority. In particular the PoA, first introduced in [32], considers the worst stable outcome (that with the highest cost), while the PoS [29] considers the best stable equilibrium (that with the lowest cost). However, we observe that in our game these two performance metrics coincide due to the uniqueness of the equilibrium reached by network users. For this reason, in the following we will refer exclusively to the first performance figure, the PoA, which has a particular importance in characterizing the efficiency of distributed game formulations. 
To determine the optimal system-wide solution, we define the social welfare $S$ as the weighted average of the delays experienced by primary and secondary users; $S$ is therefore a function of the amount $x$ of traffic sent by primary users:

$$
S(x)=\frac{\alpha x}{C-x}+\frac{\alpha(\lambda-x)}{C-\lambda}
$$

Note that $p_{1}$ and $p_{2}$ do not appear in the social welfare's expression, since all the prices paid by primary/secondary users (which represent for them a disutility or cost) correspond to a symmetric utility or gain for the Primary/Secondary operators, who collect this income in exchange for the network services they offer.

To minimize this quantity, it suffices to derive with respect to $x$ and impose its equality to zero, thus obtaining:

$$
\frac{d S(x)}{d x}=\frac{\alpha C}{(C-x)^{2}}-\frac{\alpha}{C-\lambda}=0
$$

which leads to $x_{\min }=C-\sqrt{C(C-\lambda)}$.

The optimal social welfare is therefore equal to:

$$
S\left(x_{\min }\right)=\alpha\left[\frac{C-\sqrt{C(C-\lambda)}}{\sqrt{C(C-\lambda)}}+\frac{\lambda-C+\sqrt{C(C-\lambda)}}{C-\lambda}\right]=2 \alpha\left[\sqrt{\frac{C}{C-\lambda}}-1\right] .
$$

Recall that the total traffic transmitted by primary users at the Wardrop equilibrium is given by expression (6), and the equilibrium traffic for secondary users is $\lambda_{s}=\lambda-\lambda_{p}$.

The (average) total delay experienced by primary/secondary users at equilibrium is therefore equal to:

$$
T D_{E}=\alpha \frac{\lambda_{p}}{C-\lambda_{p}}+\alpha \frac{\lambda_{s}}{C-\lambda}
$$

while the Price of Anarchy (PoA) is defined as the ratio between the cost of the worst (here, the unique) equilibrium and the social optimum, $P o A=\frac{T D_{E}}{S\left(x_{m i n}\right)}$.

Hereafter, we derive the closed-form expressions for the $P o A$ in both the considered scenarios (i.e., the TOGETHER and BEFORE scenarios). To this aim, it is sufficient to use equilibrium expressions for $\lambda_{P}$ and $\lambda_{S}$ in both scenarios. 
A. PoA for the TOGETHER scenario (the PO and SO play together)

The total delay of cognitive users at equilibrium $\left(T D_{E}^{T}\right)$ can be expressed as follows:

$$
\begin{aligned}
T D_{E}^{T} & =\alpha \frac{\lambda_{p}}{C-\lambda_{p}}+\alpha \frac{\lambda_{s}}{C-\lambda}=\frac{\alpha \lambda}{C-\lambda}-\left(p_{1}-p_{2}\right) \lambda_{p} \\
& =\frac{\alpha C(9 C-5 \lambda)-\alpha(3 C-2 \lambda) \sqrt{(C-\lambda)(9 C-5 \lambda)}}{(2 C-\lambda)[(C-\lambda)+\sqrt{(C-\lambda)(9 C-5 \lambda)}]} .
\end{aligned}
$$

Therefore, the Price of Anarchy can be calculated as:

$$
P o A_{T}=\frac{T D_{E}^{T}}{S\left(x_{\min }\right)}=\frac{C(9 C-5 \lambda) \sqrt{C-\lambda}-(3 C-2 \lambda)(C-\lambda) \sqrt{9 C-5 \lambda}}{2(2 C-\lambda)[(C-\lambda)+\sqrt{(C-\lambda)(9 C-5 \lambda)}][\sqrt{C}-\sqrt{C-\lambda}]}
$$

B. PoA for the BEFORE scenario (the PO plays before the SO)

In this case, the total delay of cognitive users at equilibrium $\left(T D_{E}^{B}\right)$ can be expressed as:

$$
\begin{aligned}
T D_{E}^{B} & =\alpha \frac{\lambda_{p}}{C-\lambda_{p}}+\alpha \frac{\lambda_{s}}{C-\lambda}=\frac{\alpha \lambda}{C-\lambda}-\left(p_{1}-p_{2}\right) \lambda_{p} \\
& =\alpha\left[-2+\frac{C}{C-\lambda}\left(Z+\frac{h}{3}\right)+\frac{1}{Z+\frac{h}{3}}\right]
\end{aligned}
$$

where

$$
Z=\left(\frac{h}{4}\right)^{1 / 3}\left[\left(\sqrt{1+\frac{4}{27} h^{2}}+1\right)^{2 / 3}+\left(\sqrt{1+\frac{4}{27} h^{2}}-1\right)^{2 / 3}\right]
$$

and $h=\frac{C-\lambda}{2 C}$.

The Price of Anarchy is therefore equal to:

$$
P o A_{B}=\frac{T D_{E}^{B}}{S\left(x_{\min }\right)}=\frac{\sqrt{C-\lambda}}{2(\sqrt{C-\lambda}-\sqrt{C})}\left[-2+\frac{C}{C-\lambda}\left(Z+\frac{h}{3}\right)+\frac{1}{Z+\frac{h}{3}}\right] .
$$

Note that both expressions (20) and (22) are independent of $\alpha$.

\section{Cognitive Users' Behavior: Replicator Dynamics}

After having characterized the static, steady-state equilibria reached by network operators and users in the joint pricing and spectrum selection game, in this section we further focus on modeling the dynamic behavior of network users. 
To this aim, we use population dynamics (and, in particular, replicator dynamics) to model the behavior of users who decide which network they should connect to, since such dynamics models network users who adapt their choices and strategies based on the observed state of the system (in terms of costs and congestion, in our case).

Before introducing replicator dynamics for our network selection game, we must first define some relevant game theoretic concepts.

\section{A. Introduction to Population Games and Replicator Dynamics}

Hereafter we briefly introduce population games and replicator dynamics; for more details, the reader is referred to the book by W. H. Sandholm [8].

1) Population Games: A population game $G$, with $Q$ non-atomic classes of players (i.e., network users) is defined by a mass and a strategy set for each class, and a payoff function for each strategy. By a non-atomic population, we mean that the contribution of each member of the population is very small; this is the case in our game, where a large set of users compete for CRN's bandwidth resources. We denote the set of classes by $\mathcal{Q}=\{1, \ldots, Q\}$, where $Q \geq 1$. The class $q$ has mass $m^{q}$. Let $S^{q}$ be the set of strategies available for players of class $q$, where $S^{q}=\left\{1, \ldots, s^{q}\right\}$. These strategies can be thought of as the actions that members of $q$ could possibly take (i.e., connecting to the primary or the secondary network).

During the game play, each player of class $q$ selects a strategy from $S^{q}$. The mass of players of class $q$ that choose the strategy $n \in S^{q}$ is denoted by $x_{n}^{q}$, where $\sum_{n \in S^{q}} x_{n}^{q}=m^{q}$. We denote the vector of strategy distributions being used by the entire population by $x=\left\{x^{1}, \ldots, x^{Q}\right\}$, where $x^{i}=\left\{x_{1}^{i}, \ldots, x_{s^{i}}^{i}\right\}$. The vector $x$ can be thought of as the state of the system.

The marginal payoff function (per mass unit) of players of class $q$ who play strategy $n$ when the state of the system is $x$ is denoted by $F_{n}^{q}(x)$, usually referred to as fitness in evolutionary game theory, which is assumed to be continuous and differentiable. The total payoff of the players of class $q$ is therefore $\sum_{n \in S^{q}} F_{n}^{q}(x) x_{n}^{q}$.

2) Replicator Dynamics: The replicator dynamics describes the behavior of a large population of agents who are randomly matched to play normal form games. It was first introduced in biology by Taylor and Jonker [33] to model the evolution of species, and it is also used in the economics field. Recently, such dynamics has been applied to many networking problems, like routing and resource allocation [34], [35]. 
Given $x_{n}^{q}$, which represents the proportion of players of class $q$ that choose strategy $n$, as illustrated before, the replicator dynamics can be expressed as follows:

$$
\dot{x}_{n}^{q}=x_{n}^{q}\left[F_{n}^{q}(x)-\frac{1}{m^{q}} \sum_{n \in S^{q}} F_{n}^{q}(x) x_{n}^{q}\right],
$$

where $\dot{x}_{n}^{q}$ represents the derivative of $x_{n}^{q}$ with respect to time.

In fact, the ratio $\dot{x}_{n}^{q} / x_{n}^{q}$ measures the evolutionary success (the rate of increase) of a strategy $n$. This ratio can be also expressed as the difference in fitness $F_{n}^{q}(x)$ of the strategy $n$ and the average fitness $\frac{1}{m^{q}} \sum_{n \in S^{q}} F_{n}^{q}(x) x_{n}^{q}$ of the class $q$.

An important concept in population games and replicator dynamics is Wardrop equilibrium [31], which we introduced in Section IV. In this context, a state $\hat{x}$ is a Wardrop equilibrium if for any class $q \in \mathcal{Q}$, all strategies being used by the members of $q$ yield the same marginal payoff to each member of $q$, whereas the marginal payoff that would be obtained by members of $q$ is lower for all strategies not used by class $q$.

\section{B. Cognitive Users' Behavior in the Network Selection Game: Replicator Dynamics}

Having reviewed the mathematical tools we will rely on, we now focus on the cognitive radio scenario illustrated in Section III, introducing replicator dynamics for the network selection game. In particular, we consider a population game $G$ with a non-atomic set of players $(q=1)$, which is defined by a strategy set denoted by $\mathcal{S}=\left\{s_{p}, s_{s}\right\}$, identical for all players, and a payoff function for each strategy; $s_{p}$ means that the player chooses the primary network, and $s_{s}$ that the player chooses the secondary network, using the spectrum holes left free by primary users.

Our goal is to determine the dynamic network selection settings $\left(X_{P}\right.$ and $\left.X_{S}=1-X_{P}\right)$, i.e., the fraction of players that choose the primary and secondary network, respectively, based on the equilibrium prices set by Primary and Secondary operators. Hence, the total traffic accepted in the primary network is equal to $\lambda_{P}=\lambda X_{P}$, and the one accepted in the secondary network is $\lambda_{S}=\lambda X_{S}$.

The proposed replicator dynamics provides a means to analyze how players can "learn" about their environment, and converge towards an equilibrium choice. Replicator dynamics is also useful to investigate the speed of convergence of strategy adaptation to reach a stable solution in the game. A mathematical analysis to bound such speed is provided in Section VII. In this case, CR users need to know some information, viz. the total cost (the service delay plus the price charged by the $\mathrm{PO} / \mathrm{SO}$, respectively) and 
the size of the populations $\left(X_{P}, X_{S}\right)$ that already performed such selection, before undertaking the best choice based on the system state.

As illustrated in Section III, the goal of each cognitive radio user is to minimize a weighted sum of his delay (latency) and price paid to the network operator (either primary or secondary), $\alpha$ being the parameter which permits to give more weight to delay with respect to the paid price. Hence, we can formalize the network selection game as follows:

$$
\begin{aligned}
\dot{X}_{P} & =K X_{P}\left[\frac{-\alpha}{C-\lambda X_{P}}-p_{1}-\left(\frac{-\alpha X_{P}}{C-\lambda X_{P}}-X_{P} \cdot p_{1}-\left(1-X_{P}\right)\left(\frac{\alpha}{C-\lambda}+p_{2}\right)\right)\right]= \\
& =K X_{P}\left(1-X_{P}\right)\left[-p_{1}+p_{2}+\frac{\alpha}{C-\lambda}-\frac{\alpha}{C-\lambda X_{P}}\right]
\end{aligned}
$$

where $\dot{X}_{P}$ represents the derivative of $X_{P}$ with respect to time.

This equation has the same structure as the replicator dynamics (see equation (23)): the first term $\left(F_{n}^{q}(x) \equiv \frac{-\alpha}{C-\lambda X_{P}}-p_{1}\right)$ corresponds to the total cost (the service delay plus the price charged by the $\mathrm{PO})$ perceived by users that choose to connect to the primary network, using a $\mathrm{M}|\mathrm{M}| 1$ approximation; the second term $\left(\frac{1}{m^{q}} \sum_{n \in S^{q}} F_{n}^{q}(x) x_{n}^{q} \equiv \frac{-\alpha X_{P}}{C-\lambda X_{P}}-X_{P} \cdot p_{1}-\left(1-X_{P}\right)\left(\frac{\alpha}{C-\lambda}+p_{2}\right)\right)$ represents the average cost/delay incurred by the fraction $X_{P}$ of primary users as well as by the fraction $X_{S}$ of secondary users (recall that $p_{1}$ and $p_{2}$ are the prices charged by the Primary and Secondary operator, respectively).

In particular, the speed of variation of $X_{P}$ is proportional to the population size $X_{P}$ (via the proportionality coefficient $K$ ), which models the willingness of the population to change strategy.

A similar equation can be written for Secondary Users, thus we can express the replicator dynamics for such SUs as follows:

$$
\begin{aligned}
\dot{X}_{S} & =K X_{S}\left[\frac{-\alpha}{C-\lambda}-p_{2}-\left(\frac{-\alpha\left(1-X_{S}\right)}{C-\lambda\left(1-X_{S}\right)}-\left(1-X_{S}\right) \cdot p_{1}-X_{S}\left(\frac{\alpha}{C-\lambda}+p_{2}\right)\right)\right]= \\
& =K X_{S}\left(1-X_{S}\right)\left[p_{1}-p_{2}-\frac{\alpha}{C-\lambda}+\frac{\alpha}{(C-\lambda)+\lambda X_{S}}\right] .
\end{aligned}
$$

Obviously, by comparing these two expressions it can be verified that condition $X_{p}+X_{s}=1$ holds. It can be demonstrated [8] that Wardrop equilibria are the stationary points of equations (24) and (25). As we will show in the next section, it can be easily proved that the unique non-trivial fixed point of such dynamics coincides with the Wardrop equilibrium point of the CR users' network selection game 
already determined in Section IV.

\section{CONVERGENCE ANALYSis OF REPLICATOR DYNAMicS}

This section provides an in-depth analysis on the replicator dynamics given by $(24)^{2}$. To this end, we rewrite it in a discretized version as follows:

$$
X_{P}(t+1)=X_{P}(t)+k X_{P}(t)\left[1-X_{P}(t)\right]\left[A-\frac{1}{B-X_{P}(t)}\right]
$$

where $k=K \alpha / \lambda, A=\lambda\left(-p_{1} / \alpha+p_{2} / \alpha+\frac{1}{C-\lambda}\right)$ and $B=C / \lambda$.

The above dynamics has three fixed points, among which 0 and 1 are trivial fixed points corresponding to the case where all users either act as secondary or primary users, respectively. $X_{P}^{*}=B-1 / A$ is the only non-trivial fixed point, which is also the Wardrop equilibrium of the game; its expression is equal to $X_{P}^{*}=\frac{\lambda_{P}}{\lambda}$, where $\lambda_{P}$ is the equilibrium flow already derived for the static game in Section IV (see expression (6)).

In the subsequent analysis, we investigate the convergence of the replicator dynamics to $X_{P}^{*}$. We start by establishing the following auxiliary lemma.

Lemma 1. Under the condition that $K\left(A-\frac{1}{B-1}\right) \leq 1$, it holds that

- $X_{P}(t+1)$ is non-decreasing w.r.t. $X_{P}(t)$ for $X_{P}(t) \in\left[0, X_{P}^{*}\right)$ and non-increasing w.r.t. $X_{P}(t)$ for $X_{P}(t) \in\left(X_{P}^{*}, 1\right]$;

- $X_{P}(t+1)>X_{P}(t), \forall X_{P}(t)<X_{P}^{*}$ and $X_{P}(t+1)<X_{P}(t), \forall X_{P}(t)>X_{P}^{*}$.

Proof: The proof of the first part is straightforward by checking the derivative $\partial X_{P}(t+1) / \partial X_{P}(t)$. Specifically, it can be checked that under the condition that $K\left(A-\frac{1}{B-1}\right) \leq 1, \partial X_{P}(t+1) / \partial X_{P}(t)>0$ when $X_{P}(t) \in\left[0, X_{P}^{*}\right)$ and $\partial X_{P}(t+1) / \partial X_{P}(t)<0$ when $X_{P}(t) \in\left(X_{P}^{*}, 1\right]$. The second part follows readily from (26).

The following theorem establishes the convergence of the replicator dynamics to the non-trivial fixed point $X_{P}^{*}$.

Theorem 1. Under the condition that $K\left(A-\frac{1}{B-1}\right) \leq 1$, the replicator dynamics depicted in (26) converges to the non-trivial fixed point $X_{P}^{*}$ for any initial state $0<X_{P}(0)<1$.

\footnotetext{
${ }^{2}$ Note that the same analysis can be conducted for (25).
} 
Proof: Consider an arbitrary sequence of update steps commencing from an initial vector $X_{P}(0)$. We distinguish the following two cases:

- Case 1: $0<X_{P}(0) \leq X_{P}^{*}$. In this case (recall that $X_{P}^{*}$ is a fixed point of (26)), it follows from Lemma 1 that: (1) $X_{P}(t) \leq X_{P}^{*}, \forall t$ and (2) $X_{P}(0) \leq X_{P}(1) \leq \cdots \leq X_{P}(t-1) \leq X_{P}(t) \leq \cdots$, i.e., $X_{P}(t)$ is a non-decreasing sequence. Since $X_{P}(t)$ is also bounded by $X_{P}^{*}$, it follows that it must converge to a limit. Since there is no fixed point other than $X_{P}^{*}$ in the range $\left(0, X_{P}^{*}\right]$, this limit must be $X_{P}^{*}$.

- Case 2: $X_{P}^{*}<X_{P}(0)<1$. This case can be proved in a similar manner. In fact (recall that $X_{P}^{*}$ is a fixed point of (26)), it follows from Lemma 1 that: (1) $X_{P}(t)>X_{P}^{*}, \forall t$ and (2) $X_{P}(0) \geq X_{P}(1) \geq$ $\cdots \geq X_{P}(t-1) \geq X_{P}(t) \geq \cdots$, i.e., $X_{P}(t)$ is a non-increasing sequence. Since $X_{P}(t)$ is also bounded by $X_{P}^{*}$, it follows that it must converge to a limit. Since there is no fixed point other than $X_{P}^{*}$ in the range $\left[X_{P}^{*}, 1\right)$, this limit must be $X_{P}^{*}$.

Combining the above analysis, the replicator dynamics is ensured to converge to the non-trivial fixed point $X_{P}^{*}$ for any initial state $0<X_{P}(0)<1$.

The above theorem essentially illustrates that with a conservative strategy (i.e., small $K$ ), the replicator dynamics is ensured to converge to the Wardrop equilibrium.

Remark. The above theorem establishes the sufficient condition for the convergence of the replicator dynamics to the unique non-trivial fixed point, which is also the Wardrop equilibrium. It follows straightforwardly that under the same condition, the equilibrium is also stable in that any deviated point from it will be dragged back under the replicator dynamics. In fact, $X_{P}^{*}$ is an evolutionary stable equilibrium. Meantime, it follows from the theorem that the two trivial fixed points 0 and 1 are not stable, in the sense that any deviation from them will drag the system to $X_{P}^{*}$.

It is also worth pointing out that Theorem 1 provides only a sufficient condition for the convergence and may be too stringent in some cases.

We further investigate the stability and the convergence speed of the replicator dynamics in the following theorem, following the guidelines of [36].

Theorem 2. Under the condition that $K\left(A-\frac{1}{B-1}\right)<1$, the non-trivial fixed point $X_{P}^{*}$ is exponentially stable under the replicator dynamics depicted in (26), i.e., there exists $0 \leq k^{\prime}<1$ such that $\left|X(t)-X_{P}^{*}\right| \leq$ $\left(k^{\prime}\right)^{t}\left|X(0)-X_{P}^{*}\right| \cdot$ 
Proof: We show that the replicator dynamics $X_{P}(t) \rightarrow X_{P}(t+1)$ in (26) is a contraction.

The contraction is defined as follows: let $(X, d)$ be a metric space, $f: X \rightarrow X$ is a contraction if there exists a constant $k^{\prime} \in[0,1)$ such that $\forall x, y \in X, d(f(x), f(y)) \leq k^{\prime} d(x, y)$, where $d(x, y)=\|x-y\|=$ $\max _{i}\left\|x_{i}-y_{i}\right\|$.

To that end, note that:

$$
d(f(x), f(y))=\|f(x)-f(y)\| \leq\left\|\frac{\partial f}{\partial x}\right\| \cdot\|x-y\|=\left\|\frac{\partial f}{\partial x}\right\| d(x, y) .
$$

If the Jacobian $\left\|\frac{\partial f}{\partial x}\right\| \leq k^{\prime}$, then $f$ is a contraction.

By some algebraic operations, we can bound the Jacobian as

$$
\|J\|_{\infty}=\max _{X_{P}(t) \in(0,1)}\left|\frac{\partial X_{P}(t+1)}{\partial X_{P}(t)}\right| \leq 1-K\left(A-\frac{1}{B-1}\right) .
$$

Hence, since the condition $K\left(A-\frac{1}{B-1}\right)<1$ holds, i.e., $\|J\|_{\infty} \leq k^{\prime} \triangleq 1-K\left(A-\frac{1}{B-1}\right)<1, X_{P}^{*}$ is exponentially stable where $k^{\prime}$ is the exponential convergence speed.

\section{NUMERICAL RESULTS}

In this section, we analyze and discuss the numerical results obtained from solving our joint pricing and spectrum access game model in different cognitive radio scenarios. More in detail, we measure the sensitivity of the operators' utilities and prices, as well as users' equilibrium flows and costs, to different parameters like the total traffic $\lambda$ accepted in the network and the channel capacity $C$.

Before doing so, let us first consider an example of a primary operator utility function $\left(U_{P}\right)$. Figure 2 shows this latter as a function of the price $p_{1}$ set by the Primary Operator (the price $p_{2}$ has been fixed to the Nash equilibrium value), with $\alpha=1, C=100$ and $\lambda=10$. By simply deriving and using the second order derivative test, it can be proved that the PO's revenue has a global maximum, as illustrated in the figure, since for small $p_{1}$ values the incoming primary traffic is priced too low, resulting in a low PO revenue, while for high $p_{1}$ values few users choose the primary network, thus diminishing its profitability.

\section{A. Effect of the traffic accepted in the network $(\lambda)$}

We first consider a CRN scenario with maximum channel capacity $C=100$ and total accepted traffic $\lambda$ varying in the $[0,100]$ range. The parameter $\alpha$, which expresses the relative importance of the experienced delay with respect to the access cost, is set to 1 , unless otherwise stated. 


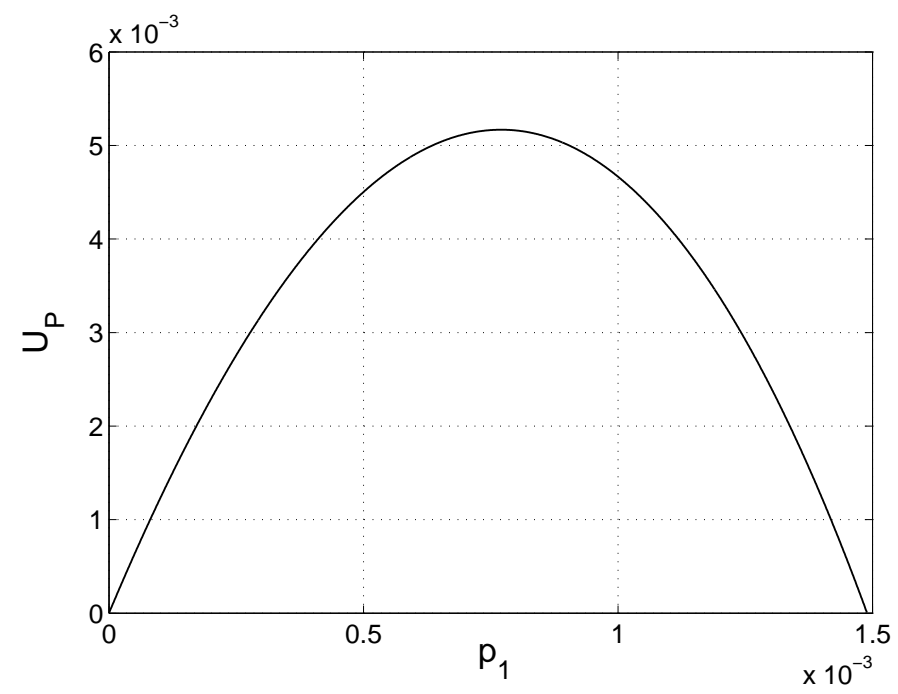

Fig. 2. Primary Operator's utility $\left(U_{P}\right)$ as a function of the imposed price $p_{1}$ in the TOGETHER scenario. Price $p_{2}$ has been fixed to the Nash equilibrium value.

Figures 3(a) and 3(b) show the prices set at the Nash equilibrium by the Primary $\left(p_{1}\right)$ and the Secondary operator $\left(p_{2}\right)$, respectively, in the two considered scenarios (the PO and SO play TOGETHER, the PO plays BEFORE the SO, anticipating the choices of this latter). The difference between the prices set by the operators in these two scenarios can be better appreciated in Figures 4(a) and 4(b) for the PO and SO, respectively. All numerical results illustrated in Figures 3 and 4 are summarized in Table IV.

It can be observed (Figure 4(a)) that in the BEFORE scenario, the PO sets a higher price than in the TOGETHER scenario, until the network is overloaded $(\lambda \leq 80)$; above this threshold, the price set by the PO in the former scenario is lower than in the latter. As for the price set by the Secondary operator (Figure 4(b)), it is always higher in the BEFORE than in the TOGETHER scenario, and such difference increases consistently for increasing $\lambda$ values. This is the reason why the PO in the BEFORE scenario can lower his price while still attracting the large majority of network users, as we will show in the following.

TABLE IV

EQUILIBRIUM PRICES $p_{1}$ AND $p_{2}$ SET BY THE PO/SO (AS WELL AS THEIR DIFFERENCE), FOR DIFFERENT VALUES OF THE TOTAL TRAFFIC $\lambda$ OFFERED TO THE NETWORK FOR BOTH THE BEFORE AND TOGETHER SCENARIOS.

\begin{tabular}{|c|c|c|c|c||c|c|}
\hline$\lambda$ & $p_{1_{\text {TOGETHER }}} \times 10^{-3}$ & $p_{1_{\text {BEFORE }}} \times 10^{-3}$ & $p_{2_{\text {TOGETHER }}} \times 10^{-3}$ & $p_{2_{\text {BEFORE }}} \times 10^{-3}$ & $\Delta p_{1} \times 10^{-3}$ & $\Delta p_{2} \times 10^{-3}$ \\
\hline 20 & 1.806 & 2.441 & 0.868 & 1.154 & 0.635 & 0.286 \\
\hline 40 & 5.242 & 6.375 & 2.374 & 2.805 & 1.133 & 0.431 \\
\hline 60 & 12.885 & 14.122 & 5.288 & 5.613 & 1.237 & 0.325 \\
\hline 80 & 37.5 & 37.5 & 12.5 & 12.5 & 0 & 0 \\
\hline 90 & 87.724 & 85.112 & 22.761 & 23.697 & -2.612 & 0.936 \\
\hline
\end{tabular}




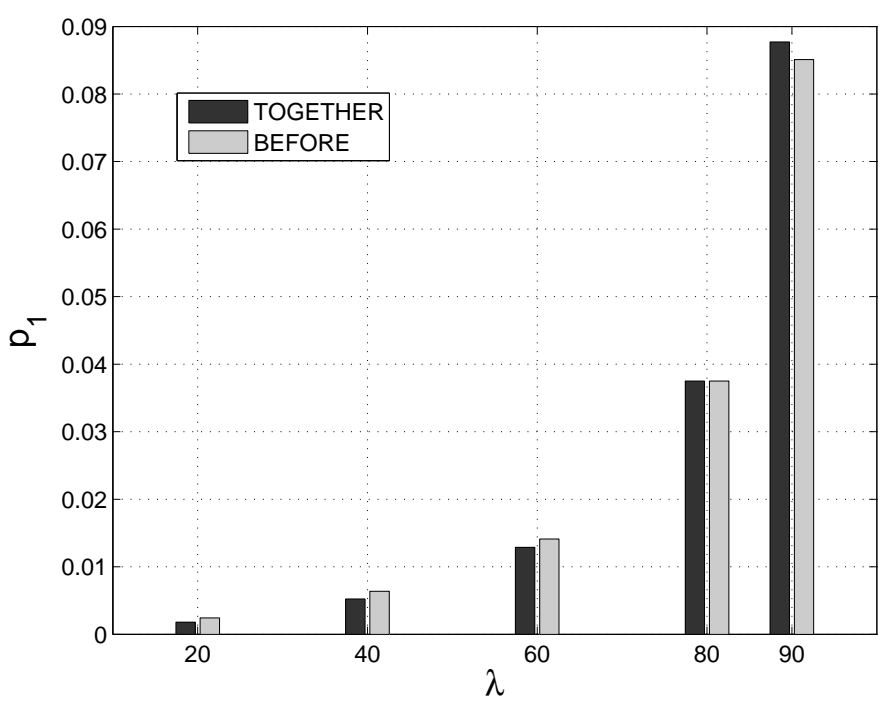

(a)

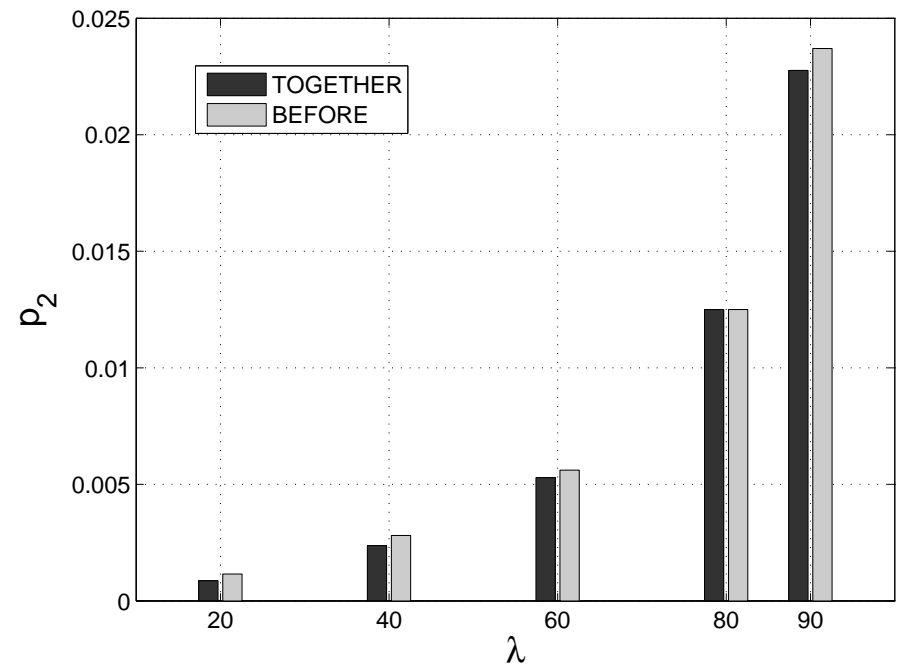

(b)

Fig. 3. (a) Equilibrium price $p_{1}$ set by the Primary operator and (b) Equilibrium price $p_{2}$ set by the Secondary operator, as a function of the total traffic $\lambda$ offered to the network for both the BEFORE and TOGETHER scenarios.

The corresponding equilibrium traffic sent by primary $\left(\lambda_{P}\right)$ and secondary users $\left(\lambda_{S}\right)$ is illustrated in Figures 5(a) and 5(b) as a function of $\lambda$, for both the considered scenarios.

We can observe that:

- The traffic accepted (and consequently, the overall fraction of users) in the primary network, $\lambda_{P}$, always increases with the offered traffic, until finally, when $\lambda \rightarrow C$, all users choose the primary network. This is due to the superior attractiveness of such network (in terms of the delay experienced 


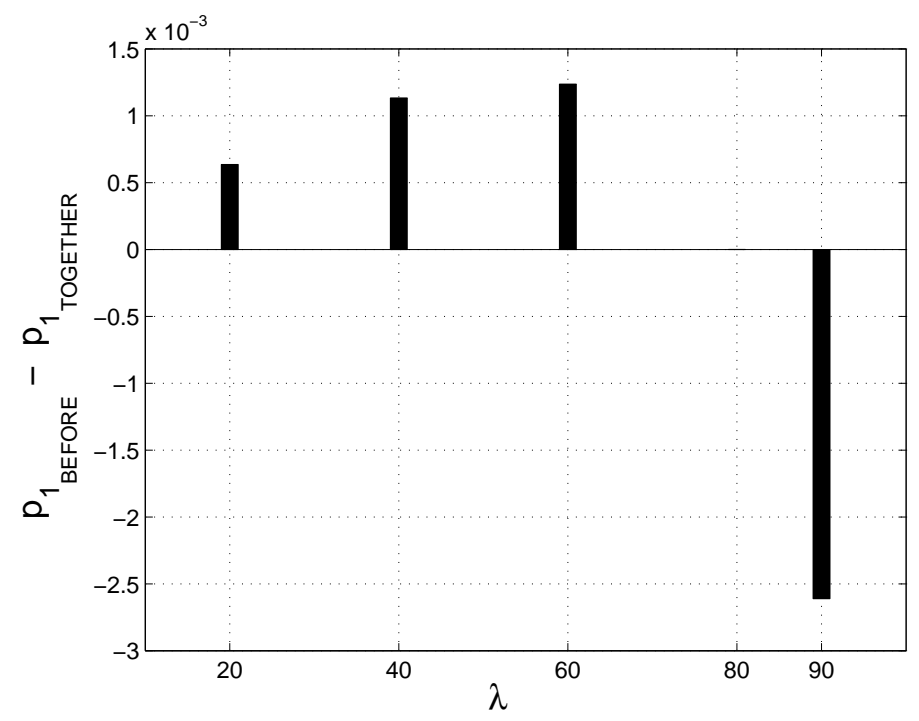

(a)

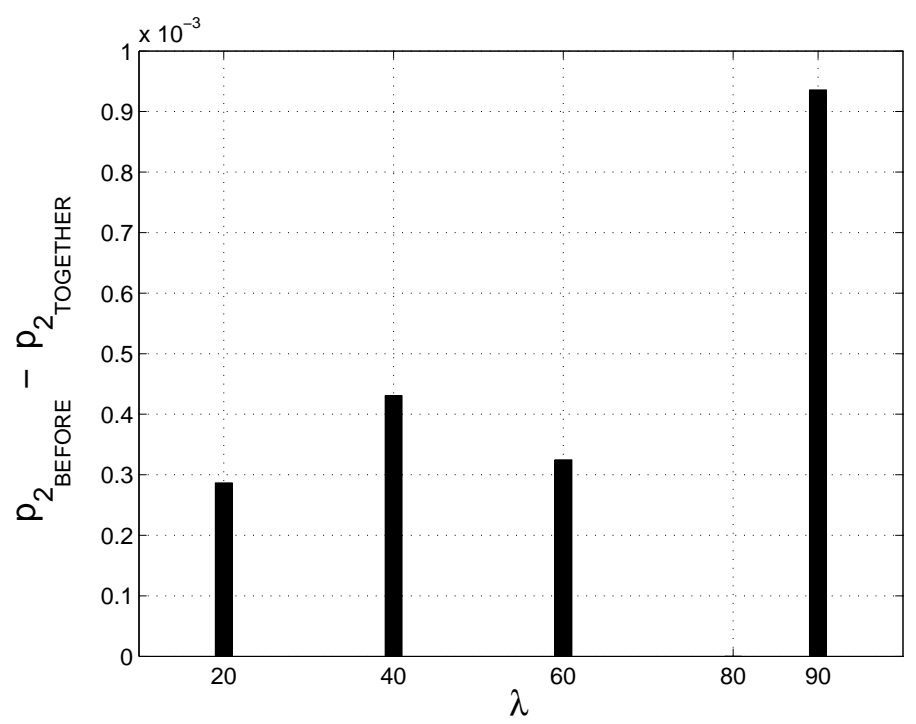

(b)

Fig. 4. (a) Difference in the equilibrium prices $p_{1}$ set by the Primary operator in the TOGETHER and BEFORE scenarios, and (b) difference in the equilibrium prices $p_{2}$ set by the Secondary operator in the same scenarios.

by users) with respect to the secondary one, since resources are licensed to primary users and SUs always observe a higher delay than PUs.

- Furthermore, concerning $\lambda_{P}$, in the BEFORE scenario the PO admits (slightly) less traffic than the SO, when $\lambda<80 \%$ of the total capacity $C$ (Figure 5(a)); this is due to the fact that the equilibrium price $p_{1}$ set by the PO in such scenario is higher than in the TOGETHER case (see Figure 4(a)), which in turn makes $\lambda_{P}$ decrease. In the high traffic regime, the PO increasingly attracts more traffic 
due to the significantly lower delay experienced in the primary network, while the SO increases $p_{2}$ in an effort to increase his utility in spite of the customer rush towards the primary network (more specifically, fewer clients choose the $\mathrm{SO}$, who reacts by raising his access price $p_{2}$ in order to increase his revenue, reaction which in turn accentuates this phenomenon).

- Concerning $\lambda_{S}$, its derivative with respect to $\lambda$ is always decreasing: it is increasingly less attractive to be a secondary user than a primary one, since for increasing $\lambda$ values the delay tends to dominate in the total cost perceived by the user.

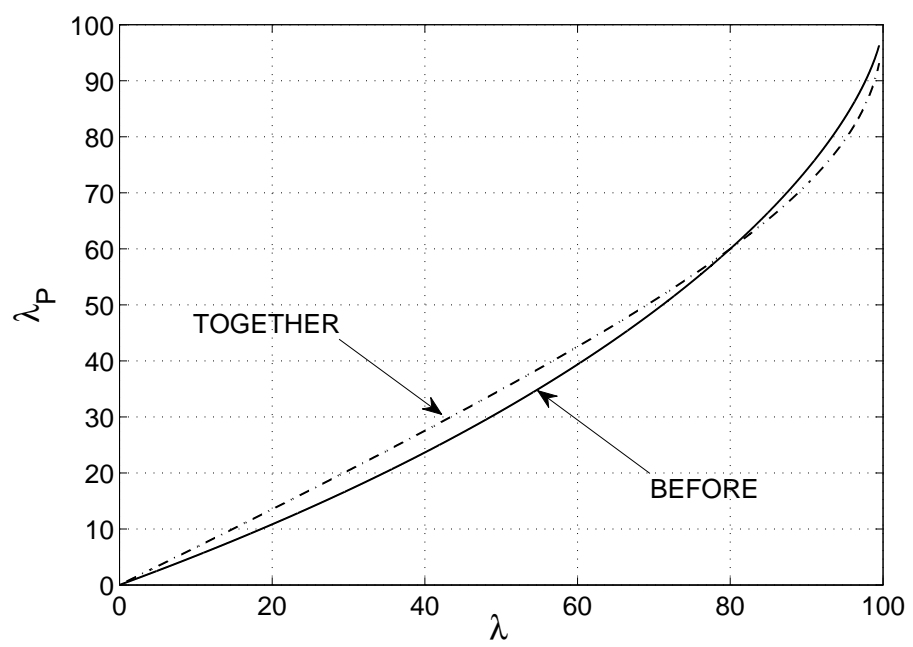

(a)

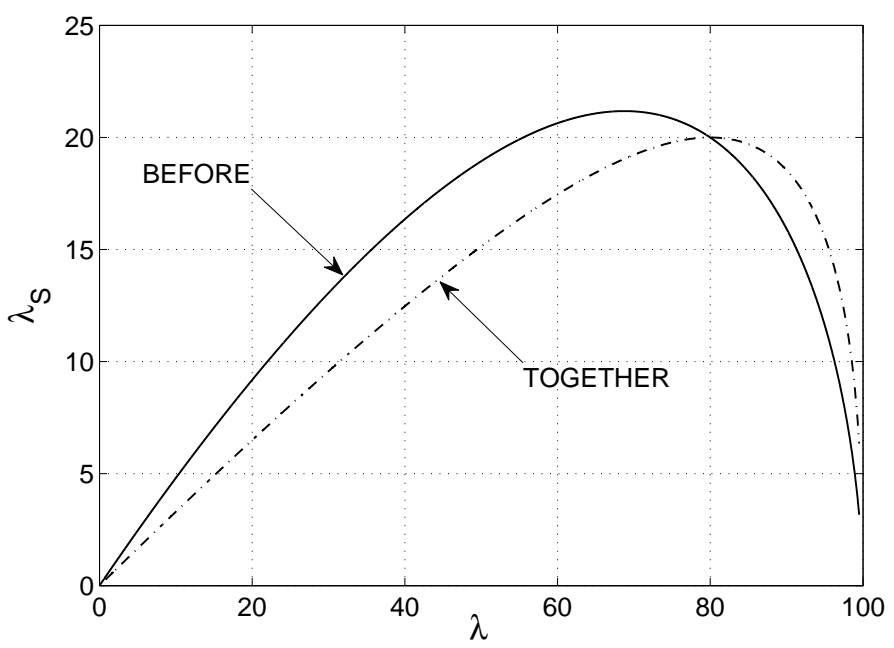

(b)

Fig. 5. Equilibrium traffic sent by primary $\left(\lambda_{P}\right)$ and secondary users $\left(\lambda_{S}\right)$ as a function of the total traffic, $\lambda$, accepted in the network, for both the TOGETHER and BEFORE scenarios.

We now focus our analysis on operators' utility, which we recall is defined as the product of the price 
set by the operator and the total flow transmitted by users that choose such operator. Figures 6(a) and 6(b) show, respectively, the difference in utilities for the Primary $\left(\Delta U_{P}\right)$ and Secondary operator $\left(\Delta U_{S}\right)$ in the TOGETHER and BEFORE scenarios.

It can be observed that it is increasingly more convenient for the PO to be a leader, anticipating the $\mathrm{SO}$, and this is reflected in the utility, which consistently grows for increasing $\lambda$ values. At the same time, for low and medium $\lambda$ values $(\lambda<0.8 C)$, even the SO obtains a higher utility in the BEFORE scenario. This means that in such scenario, both operators achieve an economic advantage at the expense of the total price paid by cognitive radio users.

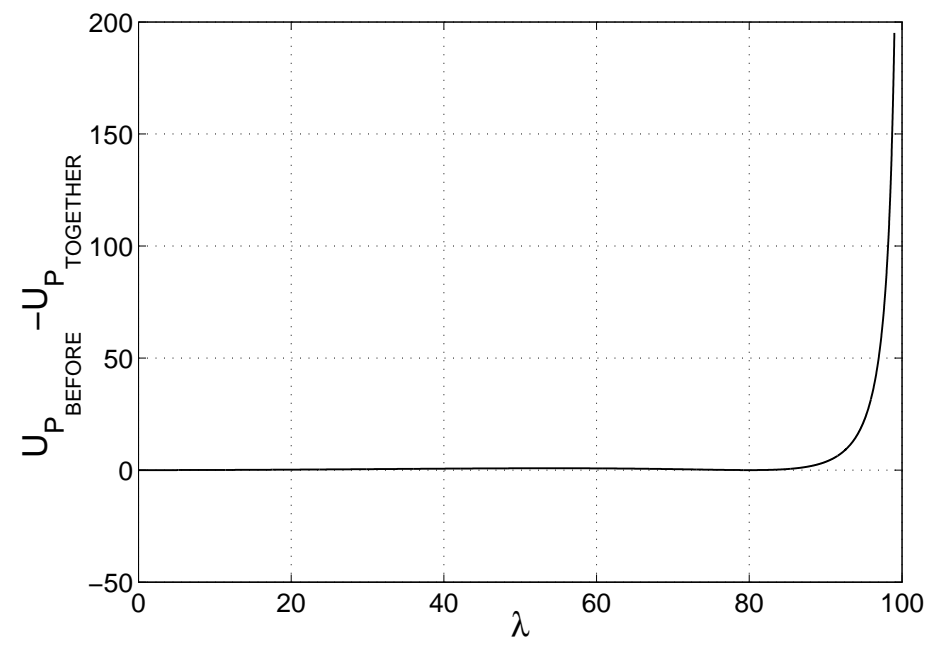

(a)

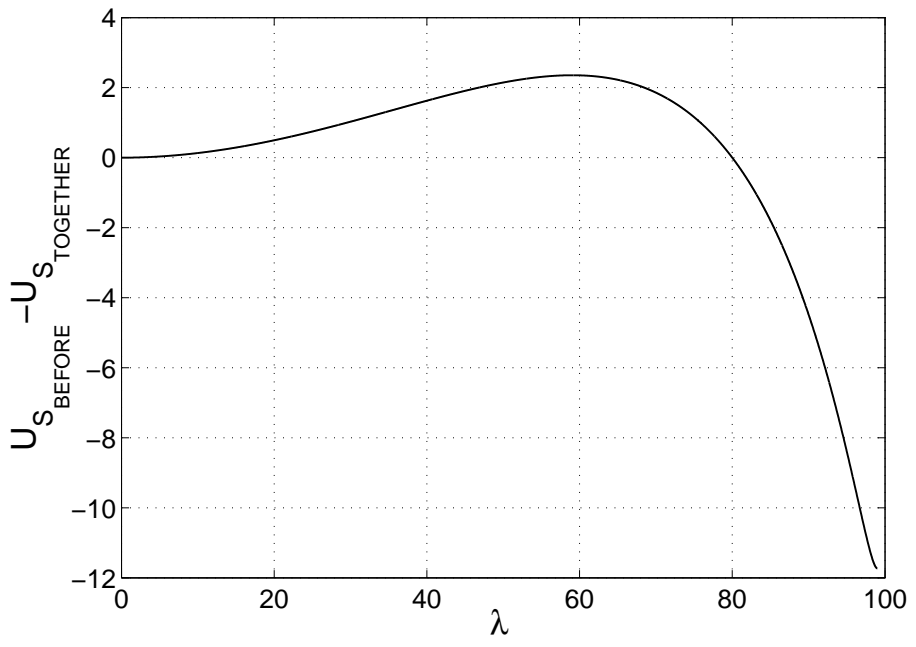

(b)

Fig. 6. (a) Difference in utilities $U_{P}$ of the Primary operator when he plays BEFORE and TOGETHER with the SO. (b) Difference in utilities $U_{S}$ of the secondary operator in the same scenarios. 


\section{B. Effect of the channel capacity $(C)$}

We now consider a variation of this network scenario, doubling the channel capacity $C$ to 200 ; the total traffic admitted in the primary network is illustrated in Figure 7. The trend is the same as already shown in Figure 5(a), and a similar behavior can be observed for the secondary traffic, which is not reported for the sake of brevity.

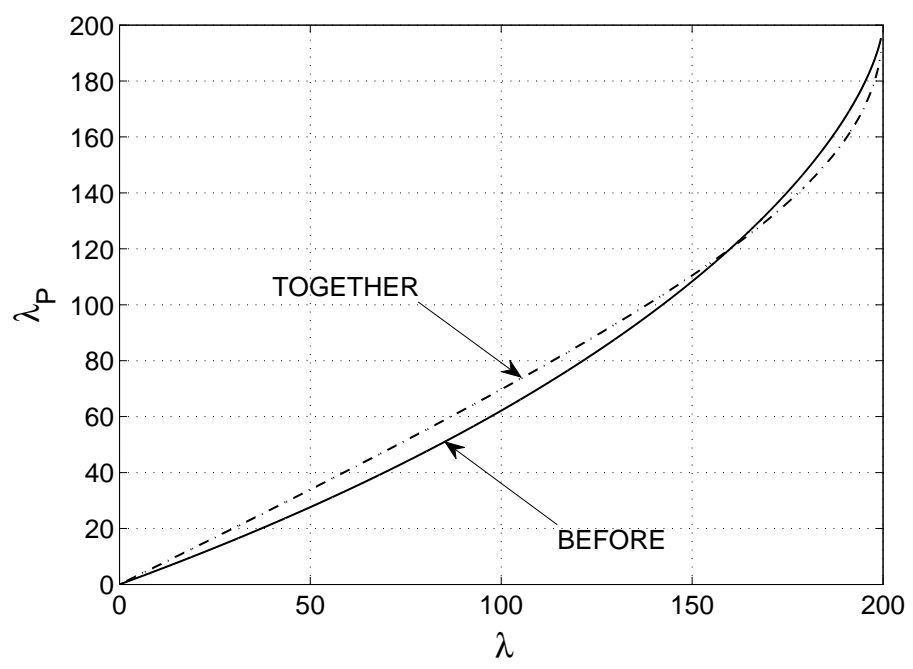

Fig. 7. Equilibrium traffic sent by primary users $\left(\lambda_{P}\right)$ as a function of the total traffic, $\lambda$, accepted in the network, for both the TOGETHER and BEFORE scenarios. The total channel capacity is $C=200$.

On the other hand, Figure 8 shows the equilibrium traffic sent by primary users as a function of the wireless channel capacity $C$, with $\lambda$ fixed to 100 . It can be observed that $\lambda_{P}$ tends to $\frac{\lambda}{2}(=50$ in this case) in the BEFORE scenario, and to $\frac{2 \lambda}{3}(\approx 66.6)$ in the TOGETHER scenario ${ }^{3}$. This behavior is in line with what already observed in Figure 7, since when $\lambda$ is consistently lower than $C$, the Primary operator who plays before the SO (BEFORE scenario) tends to admit less traffic than this latter.

We further illustrate in Figure 9 the chosen price as well as the utility perceived by the Primary operator, in both the considered scenarios, for increasing values of the channel capacity $C$ and a total accepted traffic $\lambda$ fixed to 100 (note that the prices $p_{1}$ set by the PO, illustrated in Figure 9(a), almost overlap in the two considered scenarios). A similar trend can be observed for both the price and utility of the Secondary operator (see Figure 10).

In summary, as the available capacity increases, operators fix increasingly lower prices, achieving a lower total revenue.

\footnotetext{
${ }^{3}$ It suffices to compute the limit for $C \rightarrow \infty$ of $\lambda_{P}$ in expression (6), substituting the equilibrium values $p_{1}, p_{2}$ for both the considered scenarios. Note that such limit is independent of $\alpha$.
} 


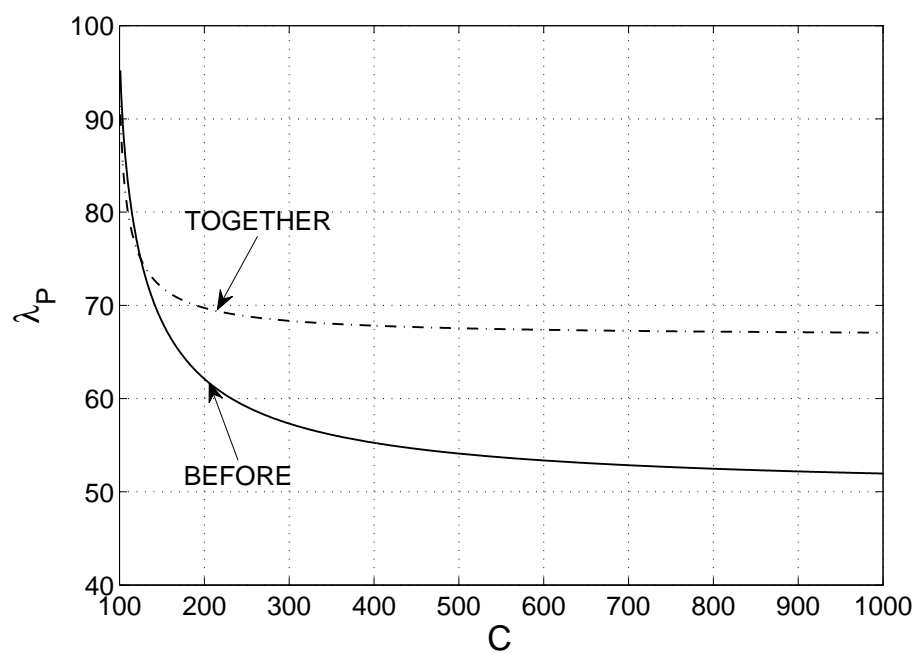

Fig. 8. Equilibrium traffic sent by primary users $\left(\lambda_{P}\right)$ as a function of the channel capacity $C$ for both the TOGETHER and BEFORE scenarios. The total traffic offered to the network, $\lambda$, is fixed and equal to 100 .

The impact of $C$ on the Price of Anarchy is further investigated in the following subsection VIII-C.

\section{Efficiency of the reached equilibria: Price of Anarchy (PoA)}

We now measure the efficiency of the equilibria reached by the system. The Price of Anarchy (PoA), which in our game coincides with the Price of Stability due to the uniqueness of the equilibria reached by operators and users, is illustrated in Figure 11 for both the TOGETHER $\left(P_{O} A_{T}\right)$ and BEFORE scenarios $\left(P o A_{B}\right)$.

When both operators play together, the PoA is equal to 1 for both extreme cases $(\lambda=0$ and $\lambda=C)$. Furthermore, it has a maximum equal to 1.0127 for $\frac{\lambda}{C}=\frac{2}{3}$, which means that, in such scenario, the equilibrium reached by the system is only $\approx 1.3 \%$ worse (in terms of the overall experienced delay) with respect to the socially optimal solution. In the BEFORE scenario, the PoA is also low, but the trend exhibited by such performance figure differs from the previous scenario, since the PoA tends to infinity for $\lambda$ approaching the channel capacity $C$. This is due to the fact that the total cost for users at equilibrium increases significantly faster than the social welfare, especially for high $\lambda$ values.

As a consequence, such situation should be avoided by market controllers either 1) by controlling the admitted traffic $\lambda$, imposing that it does not exceed a predefined fraction of the available channel capacity, or 2) by preventing the $B E F O R E$ scenario to occur, imposing antitrust policies to limit dominant position abuse. 


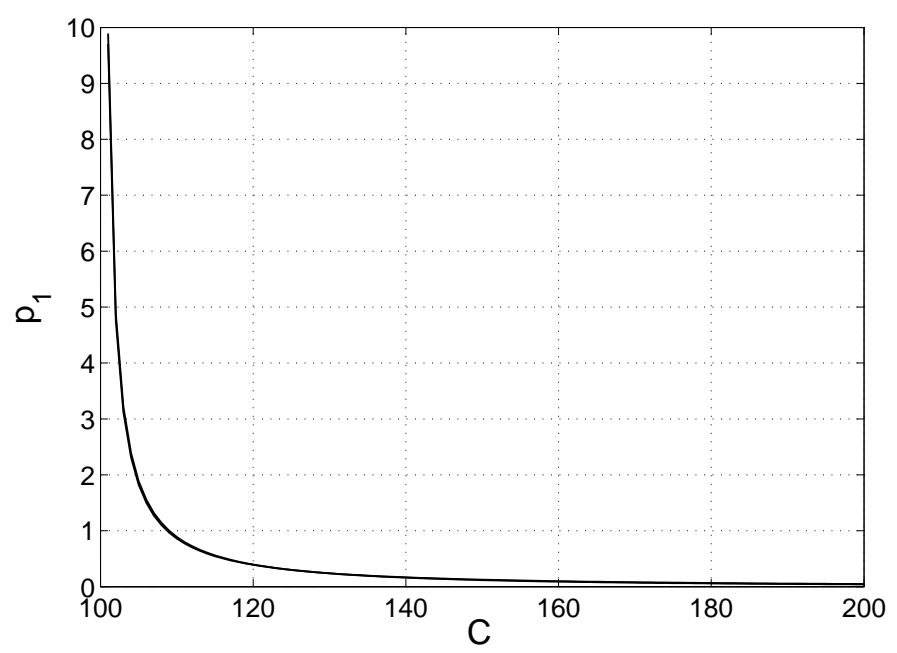

(a)

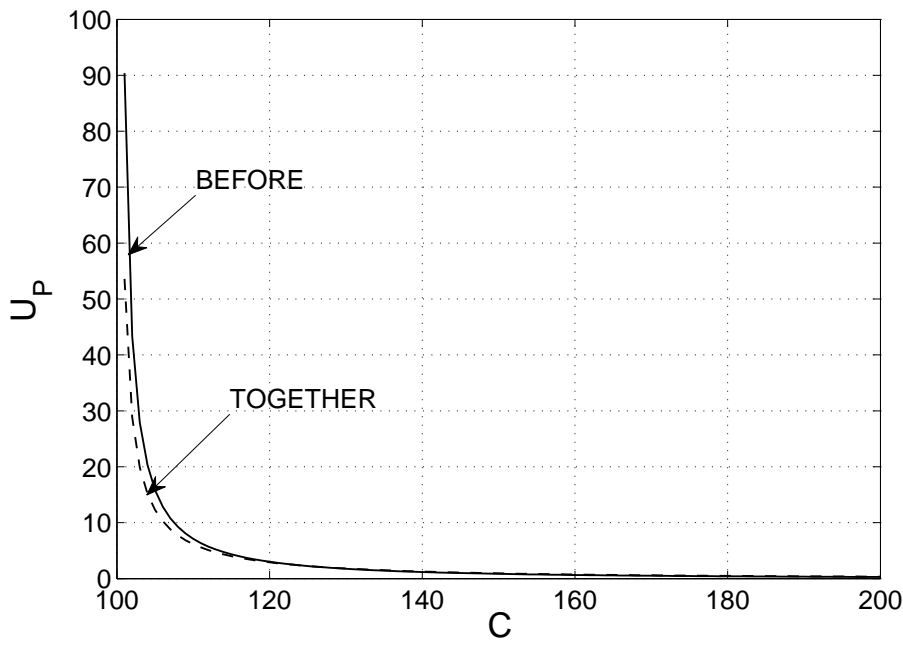

(b)

Fig. 9. (a) Primary operator's price $p_{1}$ and (b) utility $U_{P}$ as a function of the channel capacity $C$ for both the TOGETHER and BEFORE scenarios. The total traffic offered to the network, $\lambda$, is fixed and equal to 100 . Note that prices $p_{1}$ practically overlap in the two considered scenarios.

Figure 12 further reports the PoA as a function of the channel capacity $C$ for both the considered scenarios; $\lambda$ is fixed and equal to 100. It is not surprising that both curves decrease rapidly with $C$, since, as already observed in Figure 11, when $\lambda$ is consistently lower than $C$, the $\operatorname{Po} A \rightarrow 1$ in both scenarios.

In summary, we can conclude that, apart from the limiting case illustrated before for very high traffic loads, the quality of the reached equilibria is indeed excellent: when the system is loaded at less than $95 \%$, which is a reasonable operating region, the PoA is always less than 1.1, which means a loss of efficiency of $10 \%$ with respect to the social optimum. The system hence converges to a stable state which 


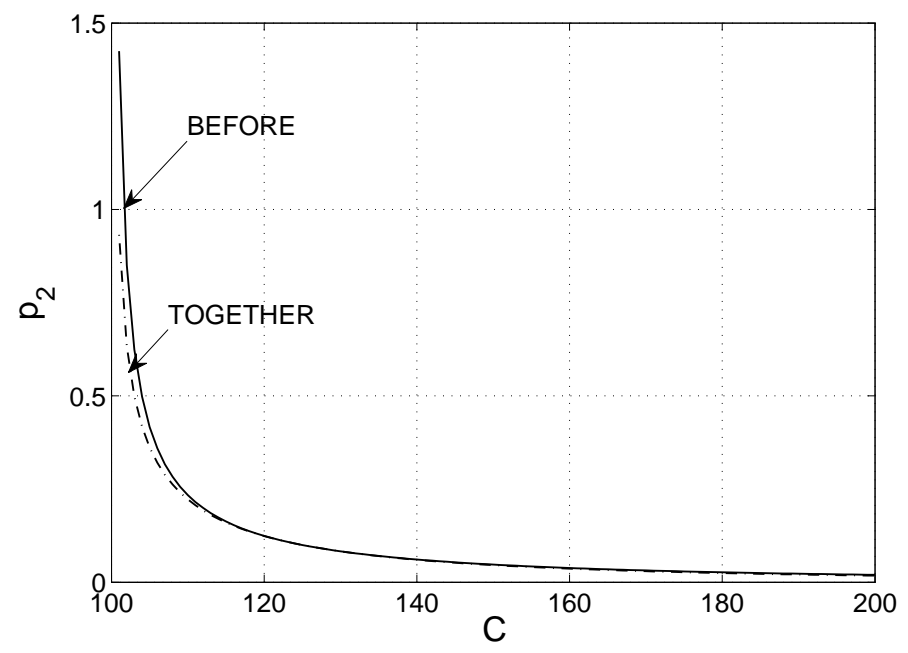

(a)

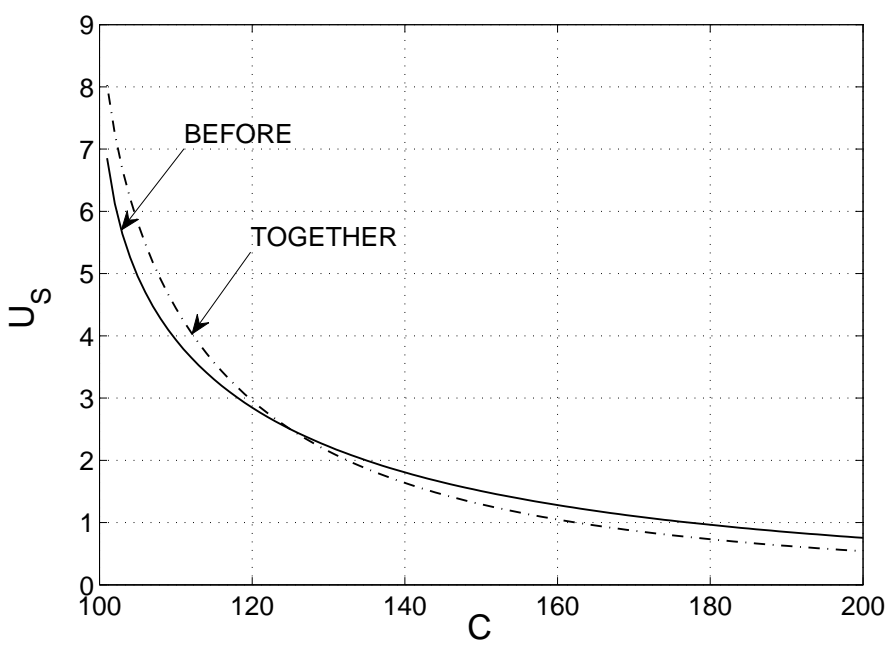

(b)

Fig. 10. (a) Secondary operator's price $p_{2}$ and (b) utility $U_{S}$ as a function of the channel capacity $C$ for both the TOGETHER and BEFORE scenarios. The total traffic offered to the network, $\lambda$, is fixed and equal to 100 .

is globally very efficient.

\section{Replicator Dynamics for the Network Selection Game}

We now analyze the convergence of the proposed replicator dynamics, fixing $\lambda=30$ and $C=100$. Figure 13 illustrates such convergence (expressed in steps needed in the replicator dynamics) of network users to a stationary solution, for different values of the parameter $K$ in equation (24), namely 1,5 and 10. More specifically, the figure reports the fraction $X_{P}$ of users that choose the primary network. We consider both cases where the initial fraction of such users is close to zero (Figure 13(a)) and one 


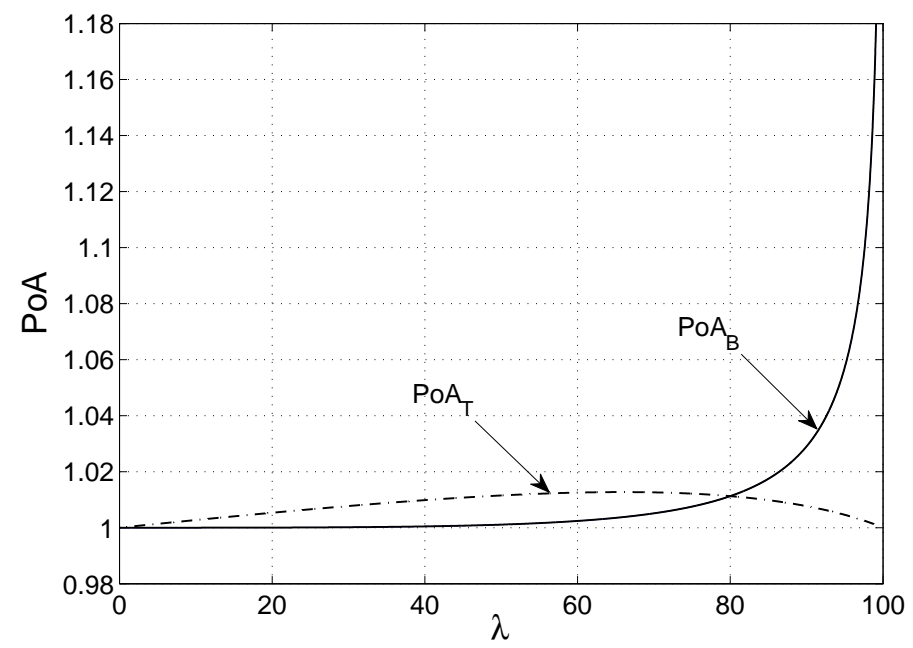

Fig. 11. The Price of Anarchy as a function of the total traffic offered to the network, $\lambda$, in both the TOGETHER $\left(P o A_{T}\right)$ and $B E F O R E\left(P o A_{B}\right)$ scenarios.

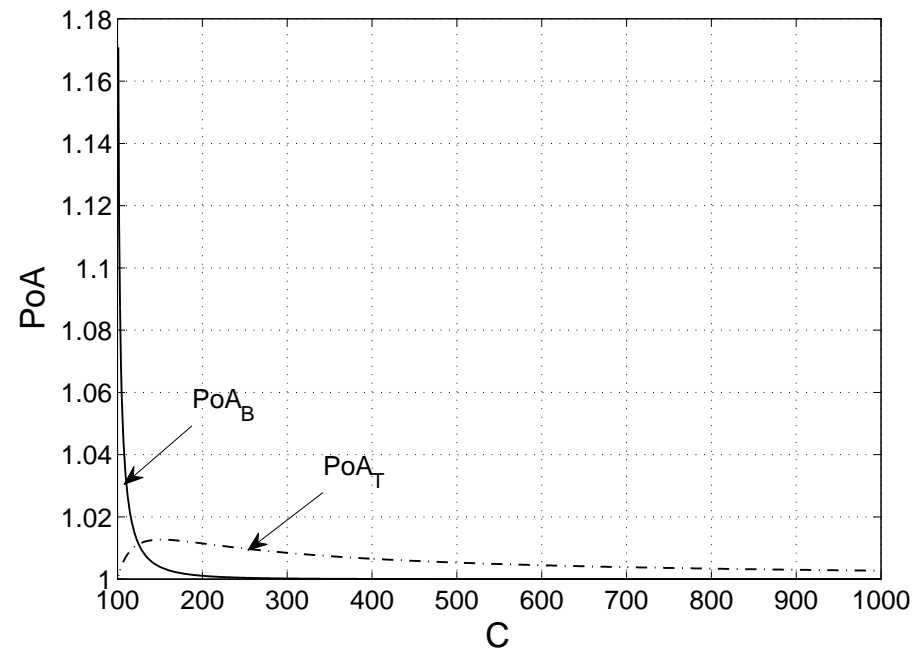

Fig. 12. The Price of Anarchy as a function of the channel capacity $C$ for both the TOGETHER $\left(P o A_{T}\right)$ and BEFORE $\left(P o A_{B}\right)$ scenarios. The total traffic offered to the network, $\lambda$, is fixed and equal to 100 .

(Figure 13(b)).

Note that the speed of convergence to the unique stable equilibrium point of the dynamics $\left(X_{P}^{*} \approx 0.68\right.$, in such scenario) increases for increasing $K$ values. Furthermore, when $p_{1}$ and $p_{2}$ are equilibrium price values, we observe that the convergence conditions demonstrated in Theorems 1 and 2 for our proposed replicator dynamics (see the previous section) are always satisfied. 


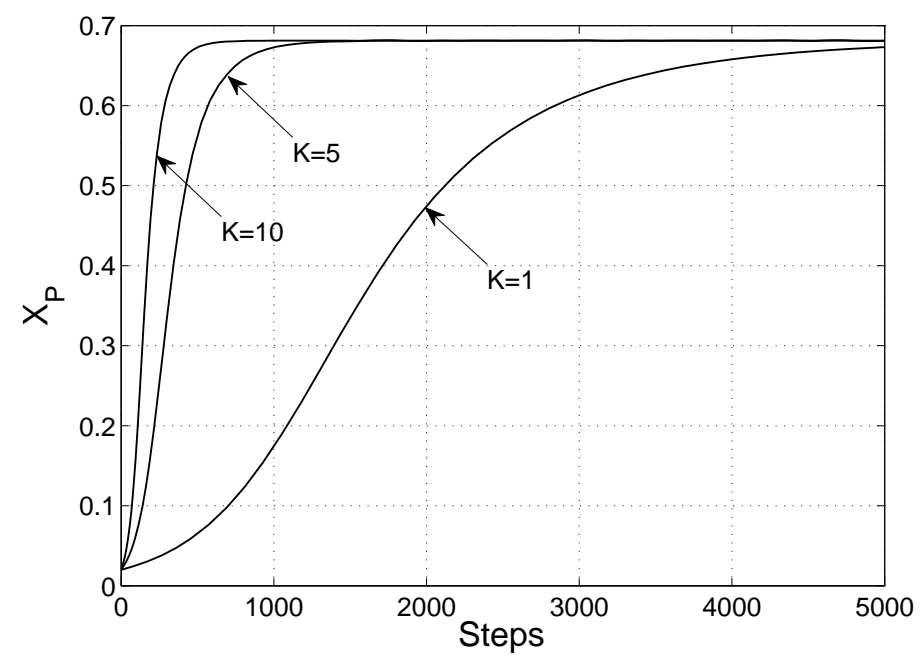

(a)

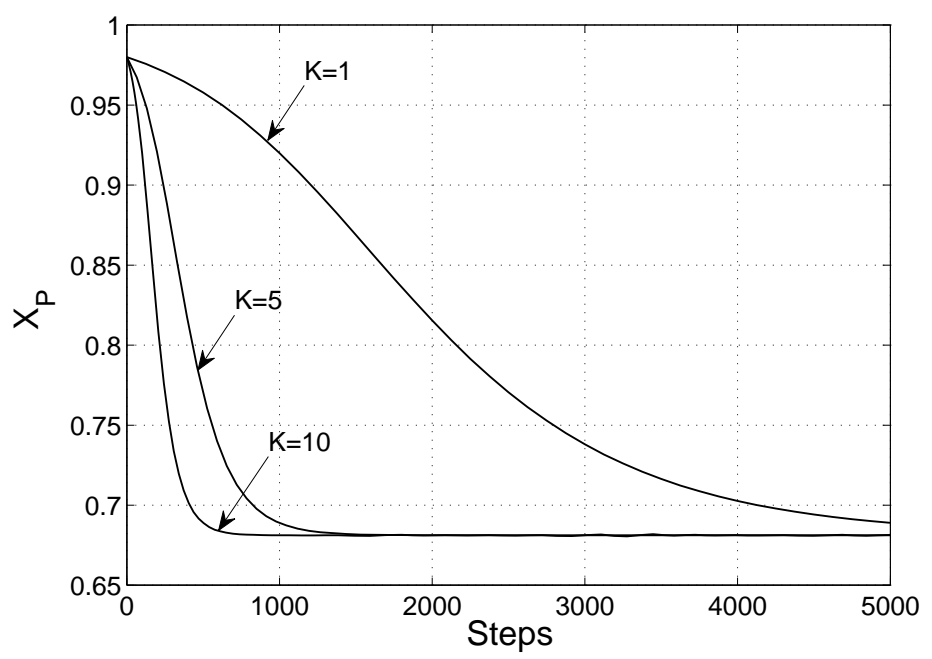

(b)

Fig. 13. Convergence of Primary Users to the stationary point $\left(X_{P}^{*} \approx 0.68\right)$. The initial point is (a) lower or (b) higher than the equilibrium.

\section{CONCLUSION}

In this paper, we tackled a fundamental problem related to Cognitive Radio Networks, i.e., the joint pricing and Primary/Secondary network selection process. More specifically, we considered a CRN scenario which is composed of primary/secondary networks and a set of Cognitive Radio users who must decide whether to subscribe to the primary network for guaranteed bandwidth or to access the secondary network, paying a lower price at the expense of possible service degradation (in terms of experienced delay and congestion). At the same time, we studied the pricing game between the Primary 
and Secondary operators, considering two practical cases where such operators fix their access price simultaneously, and where the PO anticipates the SO strategy, exploiting his dominant position.

We computed optimal, stable pricing values and network selection settings; furthermore, we studied network users' dynamics using a population game model, and we determined its convergence properties under replicator dynamics. Numerical results demonstrate that our game model captures the main factors behind cognitive network pricing and access network selection, thus representing a promising framework for the design and understanding of cognitive radio systems.

A key finding of the present study is that the advantage for the PO to play before the SO can be significant, especially in a high traffic regime; this has an adverse impact on customers' choices, since in such situation the equilibria reached by cognitive radio users drift away from the social optimum, and the Price of Anarchy tends to infinity. It is therefore important (e.g., for government, regulation authorities), to implement actions that prevent or limit such dominant position abuse, if possible.

Apart from this limiting case, which occurs exclusively for very high traffic regimes, we observe that the quality of the reached equilibria is excellent: when the system is loaded at less than 95\%, which seems a reasonable operating region, the PoA is always less than 1.1 (regardless of the order in which operators fix their price), which means a loss of efficiency of $10 \%$ with respect to the social optimum. Hence, the system is guaranteed to converge to a stable state which is very efficient from a social point of view.

\section{ACKNOWLEDGMENTS}

This work was partially supported by ANR in the framework of the ANR Green-Dyspan project.

\section{REFERENCES}

[1] I.F. Akyildiz, W.Y. Lee, M.C. Vuran, and S. Mohanty. A survey on spectrum management in cognitive radio networks. IEEE Communications Magazine, pages 40-48, vol. 46, no. 4, April 2008.

[2] J. Elias, F. Martignon, A. Capone, and E. Altman. Non-cooperative spectrum access in cognitive radio networks: a game theoretical model. Elsevier Computer Networks, pages 3832-3846, vol. 55, no. 17, December 2011.

[3] K. Jagannathan, I. Menache, E. Modiano, and G. Zussman. Non-cooperative spectrum access-the dedicated vs. free spectrum choice. IEEE Journal on Selected Areas in Communications, 30(11):2251-2261, December 2012.

[4] S. Wang, J. Zhang, and L. Tong. Delay Analysis for Cognitive Radio Networks with Random Access: A Fluid Queue View. In Proc. of IEEE Infocom 2010, San Diego, CA, USA, March 2010.

[5] Q. Zhao, L. Tong, A. Swami, and Y. Chen. Decentralized cognitive MAC for opportunistic spectrum access in ad hoc networks: A POMDP framework. IEEE Journal on Selected Areas in Communications, 25(3):589-600, 2007. 
[6] X. Li, Q. Zhao, X. Guan, and L. Tong. On the performance of cognitive access with periodic spectrum sensing. In Proceedings of the ACM workshop on Cognitive radio networks, pages 31-36, Beijing, China, September 20-25, 2009.

[7] O. Habachi and Y. Hayel. Optimal opportunistic sensing in cognitive radio networks. IET Commun., 6(8):797-804, 2012.

[8] W. H. Sandholm. Population Games and Evolutionary Dynamics. MIT press, January 2011.

[9] T.L. Vincent and J.S. Brown. Evolutionary game theory, natural selection, and Darwinian dynamics. Cambridge University Press, 2005.

[10] E. Altman, T. Boulogne, R. El-Azouzi, T. Jimenez, and L. Wynter. A survey on networking games in telecommunications. Elsevier Computers \& Operations Research, 33(2):286-311, 2006.

[11] Y.C. Liang, K.C. Chen, G.Y. Li, and P. Mahonen. Cognitive radio networking and communications: An overview. IEEE Transactions on Vehicular Technology, 60(7):3386-3407, 2011.

[12] G.S. Kasbekar and S. Sarkar. Spectrum pricing games with spatial reuse in cognitive radio networks. IEEE Journal on Selected Areas in Communications, 30(1):153-164, 2012.

[13] D. Xu, X. Liu, and Z. Han. Decentralized bargain: A two-tier market for efficient and flexible dynamic spectrum access. IEEE Transactions on Mobile Computing, June 2012.

[14] K. Zhu, D. Niyato, P. Wang, and Z. Han. Dynamic spectrum leasing and service selection in spectrum secondary market of cognitive radio networks. IEEE Transactions on Wireless Communications, 11(3):1136-1145, 2012.

[15] R. Xie, F. R. Yu, and H. Ji. Dynamic resource allocation for heterogeneous services in cognitive radio networks with imperfect channel sensing. IEEE Transactions on Vehicular Technology, 61(2):770-780, 2012.

[16] T. Elkourdi and O. Simeone. Spectrum leasing via cooperation with multiple primary users. IEEE Transactions on Vehicular Technology, 61(2):820-825, 2012.

[17] Y. Li, S. K. Jayaweera, M. Bkassiny, and K. A. Avery. Optimal myopic sensing and dynamic spectrum access in cognitive radio networks with low-complexity implementations. IEEE Trans. on Wireless Commun., 11(7):2412-2423, 2012.

[18] W. Saad, Z. Han, H.V. Poor, T. Basar, and J.B. Song. A cooperative bayesian nonparametric framework for primary user activity monitoring in cognitive radio networks. IEEE J. Sel. Areas Commun., 30(9):1815-1822, 2012.

[19] D. Niyato and E. Hossain. Dynamics of network selection in heterogeneous wireless networks: an evolutionary game approach. IEEE Transactions on Vehicular Technology, pages 2008-2017, vol. 58, no. 4, 2009.

[20] D. Niyato, E. Hossain, and Z. Han. Dynamics of Multiple-Seller and Multiple-Buyer Spectrum Trading in Cognitive Radio Networks: A Game-Theoretic Modeling Approach. IEEE Trans. Mobile Comput., pages 1009-1022, vol. 8, no. 8, 2009.

[21] X. Chen and J. Huang. Evolutionarily stable spectrum access. IEEE Transactions on Mobile Computing, April 2012.

[22] S. Iellamo, L. Chen, and M. Coupechoux. Imitation-based spectrum access policy for csma/ca-based cognitive radio networks. In Proc. of IEEE Wireless Communications and Networking Conference (WCNC 2012), pages 2780-2785, Paris, France, April 1-4, 2012.

[23] X. Chen and J. Huang. Spatial spectrum access game: Nash equilibria and distributed learning. In Proceedings of the thirteenth ACM international symposium on Mobile Ad Hoc Networking and Computing (MobiHoc 2012), pages 205-214, Hilton Head Island, South Carolina, USA, June 11-14, 2012.

[24] J. Elias, F. Martignon, and E. Altman. Joint Pricing and Cognitive Radio Network Selection: a Game Theoretical Approach. In Proc. of WiOpt 2012, Paderborn, Germany, May 2012. 
[25] J. Elias and F. Martignon. Joint Spectrum Access and Pricing in Cognitive Radio Networks with Elastic Traffic. In Proc. of IEEE International Conference on Communications, ICC'10, Cape Town, South Africa, May, 2010.

[26] S. Jafar and S. Srinivasa. Capacity limits of cognitive radio with distributed and dynamic spectral activity. IEEE Journal on Selected Areas in Communications, 25(3):529-537, 2007.

[27] S. Srinivasa and S. Jafar. Cognitive radios for dynamic spectrum access-the throughput potential of cognitive radio: A theoretical perspective. IEEE Communications Magazine, 45(5):73-79, 2007.

[28] S. Srinivasa and S. Jafar. How much spectrum sharing is optimal in cognitive radio networks? IEEE Transactions on Wireless Communications, 7(10):4010-4018, 2008.

[29] E. Anshelevich, A. Dasgupta, J. Kleinberg, E. Tardos, T. Wexler, and T. Roughgarden. The price of stability for network design with fair cost allocation. In Proc. of the 45th Annual Symposium on Foundations of Computer Science, pages 295-304, Rome, Italy, October 17-19, 2004.

[30] S. Albers, S. Eilts, E. Even-Dar, Y. Mansour, and L. Roditty. On nash equilibria for a network creation game. In Proceedings of the 17th annual ACM-SIAM symposium on Discrete algorithm, pages 89-98, Miami, Florida, USA, January 2006.

[31] J.G. Wardrop. Some theoretical aspects of road traffic research. In Proc. of the Institute of Civil Engineers, part II, pages 325-378, vol. 1, 1952.

[32] E. Koutsoupias and C. Papadimitriou. Worst-case equilibria. In Proc. of the 16th Annual Symposium on Theoretical Aspects of Computer Science, pages 404-413, Trier, Germany, March 4-6, 1999.

[33] P.D. Taylor and L.B. Jonker. Evolutionary stable strategies and game dynamics. Mathematical Biosciences, pages 145-156, vol. 40, no. 1-2, 1978.

[34] S. Shakkottai, E. Altman, and A. Kumar. Multihoming of users to access points in WLANs: A population game perspective. IEEE Journal on Selected Areas in Communications, pages 1207-1215, vol. 25, no. 6, 2007.

[35] E. Altman, Y. Hayel, and H. Kameda. Evolutionary dynamics and potential games in non-cooperative routing. In Proc. of WiOpt 2007, pages 1-5, Limassol, Cyprus, April 16-20, 2007.

[36] D. Niyato and E. Hossain. Competitive spectrum sharing in cognitive radio networks: a dynamic game approach. IEEE Transactions on Wireless Communications, pages 2651-2660, vol. 7, no. 7, 2008. 